\title{
Hubble PanCET: an extended upper atmosphere of neutral hydrogen around the warm Neptune GJ 3470b
}

\author{
V. Bourrier ${ }^{1}$, A. Lecavelier des Etangs ${ }^{2}$, D. Ehrenreich ${ }^{1}$, J. Sanz-Forcada $^{3}$, R. Allart ${ }^{1}$, G. E. Ballester ${ }^{4}$, \\ L. A. Buchhave ${ }^{5}$, O. Cohen $^{6}$, D. Deming ${ }^{7}$, T. M. Evans $^{8}$, A. García Muñoz ${ }^{9}$, G. W. Henry ${ }^{10}$, T. Kataria ${ }^{11}$, \\ P. Lavvas $^{12}$, N. Lewis ${ }^{13}$, M. López-Morales ${ }^{14}$, M. Marley ${ }^{15}$, D. K. Sing ${ }^{16,17}$, and H. R. Wakeford ${ }^{18}$ \\ ${ }^{1}$ Observatoire de l'Université de Genève, 51 chemin des Maillettes, 1290 Sauverny, Switzerland \\ e-mail: vincent.bourrier@unige.ch \\ ${ }^{2}$ Institut d'Astrophysique de Paris, CNRS, UMR 7095 \& Sorbonne Universités, UPMC Paris 6, 98 bis bd Arago, 75014 Paris, \\ France \\ ${ }^{3}$ Centro de Astrobiologia (CSIC-INTA), ESAC Campus, PO Box 78, 28691 Villanueva de la Canada, Madrid, Spain \\ ${ }^{4}$ Department of Planetary Sciences \& Lunar \& Planetary Laboratory, University of Arizona, 1541 E Univ. Blvd., Tucson, AZ 85721 , \\ USA \\ ${ }^{5}$ DTU Space, National Space Institute, Technical University of Denmark, Elektrovej 328, 2800 Kgs. Lyngby, Denmark \\ ${ }^{6}$ Lowell Center for Space Science and Technology, University of Massachusetts, Lowell, Massachusetts 01854, USA \\ ${ }^{7}$ Department of Astronomy, University of Maryland, College Park, MD 20742, USA \\ ${ }^{8}$ Astrophysics Group, Physics Building, University of Exeter, Stocker Road, Exeter EX4 4QL, UK \\ ${ }^{9}$ Zentrum für Astronomie und Astrophysik, Technische Universität Berlin, 10623 Berlin, Germany \\ ${ }^{10}$ Center of Excellence in Information Systems, Tennessee State University, Nashville, TN 37209, USA \\ ${ }^{11}$ NASA Jet Propulsion Laboratory, 4800 Oak Grove Dr, Pasadena, CA 91109, USA \\ ${ }^{12}$ Groupe de Spectroscopie Moléculaire et Atmosphérique, Université de Reims, Champagne-Ardenne, CNRS UMR 7331, France \\ ${ }^{13}$ Department of Astronomy and Carl Sagan Institute, Cornell University, 122 Sciences Drive, 14853, Ithaca, NY, USA \\ ${ }^{14}$ Harvard-Smithsonian Center for Astrophysics, 60 Garden Street, Cambridge, MA 02138, USA \\ ${ }^{15}$ NASA Ames Research Center, Moffett Field, CA, USA \\ ${ }^{16}$ Department of Physics and Astronomy, University of Exeter, Exeter, UK \\ ${ }^{17}$ Department of Earth and Planetary Sciences, Johns Hopkins University, Baltimore, MD, USA \\ ${ }^{18}$ Space Telescope Science Institute, 3700 San Martin Drive, Baltimore, MD 21218, USA
}

Received 19 June 2018 / Accepted 4 October 2018

\begin{abstract}
GJ 3470b is a warm Neptune transiting an M-dwarf star at the edge of the evaporation desert. It offers the possibility of investigating how low-mass, close-in exoplanets evolve under the irradiation from their host stars. We observed three transits of GJ 3470b in the Lyman- $\alpha$ line with the Hubble Space Telescope (HST) as part of the Panchromatic Comparative Exoplanet Treasury (PanCET) program. Absorption signatures are detected with similar properties in all three independent epochs, with absorption depths of $35 \pm 7 \%$ in the blue wing of the line, and $23 \pm 5 \%$ in the red wing. The repeatability of these signatures, their phasing with the planet transit, and the radial velocity of the absorbing gas allow us to conclude that there is an extended upper atmosphere of neutral hydrogen around GJ 3470b. We determine from our observations the stellar radiation pressure and XUV irradiation from GJ 3470 and use them to perform numerical simulations of the upper atmosphere of GJ 3470b with the EVaporating Exoplanets (EVE) code. The unusual redshifted signature can be explained by the damping wings of dense layers of neutral hydrogen that extend beyond the Roche lobe and are elongated in the direction of the planet motion. This structure could correspond to a shocked layer of planetary material formed by the collision of the expanding thermosphere with the wind of the star. The blueshifted signature is well explained by neutral hydrogen atoms escaping at rates of about $10^{10} \mathrm{~g} \mathrm{~s}^{-1}$ that are blown away from the star by its strong radiation pressure and are quickly photoionized, resulting in a smaller exosphere than that of the warm Neptune GJ 436b. The stronger escape from GJ 3470b, however, may have led to the loss of about $4-35 \%$ of its current mass over its $\sim 2$ Gyr lifetime.
\end{abstract}

Key words. techniques: spectroscopic - planets and satellites: atmospheres - planets and satellites: individual: GJ 3470b planet-star interactions - ultraviolet: stars

\section{Introduction}

Extended exospheres were first detected around strongly irradiated gas giants through transit observations in the ultraviolet spectrum of their host stars. The hot Jupiters HD 209458b (Vidal-Madjar et al. 2003, 2004, 2008; Ehrenreich et al. 2008; Ben-Jaffel \& Sona Hosseini 2010) and HD 189733b (Lecavelier des Etangs et al. 2010, 2012; Bourrier et al. 2013) revealed Lyman- $\alpha$ transit absorption depths about 10 times larger than their planetary transit at optical wavelengths. Absorption signatures measured in the blue wing of the Lyman- $\alpha$ line traced the presence of neutral hydrogen gas beyond the gravitational influence of the planets and revealed that it moves away from the stars at velocities reaching $\sim 100-200 \mathrm{~km} \mathrm{~s}^{-1}$. This evaporation arises from the deposition of stellar X-ray and extreme ultraviolet radiation (XUV) at the base of the planet 


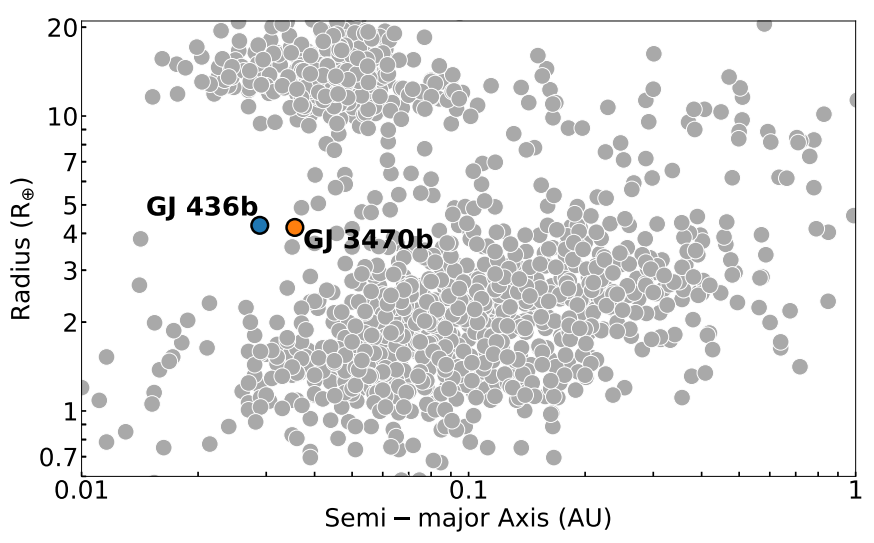

Fig. 1. Distribution of planetary radius as a function of distance to the star. Physical properties of exoplanets were extracted from the Extrasolar Planets Encyclopaedia (exoplanet.eu) in April 2018. Evaporation shaped the population of hot close-in planets, likely resulting in the formation of a desert of hot planets with intermediate size. The warm Neptunes GJ 3470b and GJ 436b lie at the edge of this desert.

thermosphere, leading to its hydrodynamic expansion up to altitudes of several planetary radii (e.g., Vidal-Madjar et al. 2003; Lammer et al. 2003; Lecavelier des Etangs et al. 2004; García Muñoz 2007; Johnstone et al. 2015; Guo \& Ben-Jaffel 2016).

Despite tremendous mass losses on the order of thousands of tons per second, hot Jupiters are massive enough for evaporation to only weakly affect their long-term evolution (e.g., Yelle 2004; Lecavelier des Etangs 2007; Hubbard et al. 2007; Ehrenreich \& Désert 2011). However, a dearth of exoplanets exists at small orbital distances (Fig. 1), ranging from sub-Jupiters (Lecavelier des Etangs 2007; Davis \& Wheatley 2009; Szabó \& Kiss 2011; Mazeh et al. 2016) to super-Earths (Beaugé \& Nesvorný 2013; Sanchis-Ojeda et al. 2014; Lundkvist et al. 2016; Fulton et al. 2017; Fulton \& Petigura 2018). Theoretical studies suggest that atmospheric escape plays a major role in shaping this so-called desert (e.g., Lopez et al. 2012; Lopez \& Fortney 2013; Jin et al. 2014; Kurokawa \& Nakamoto 2014) and valley (Owen \& Wu 2017; Jin \& Mordasini 2018) in the exoplanet population, with strongly irradiated, low-density planets that are unable to retain their escaping gaseous atmosphere. Some of the very hot rocky super-Earths could thus be the evaporated remnants of more massive planets ("chthonian" planets; Lecavelier des Etangs et al. 2004).

In this context, it is essential to observe exoplanets at the borders of the desert of hot, intermediate-size planets (Fig. 1) to understand the origin of this structure and how planetary and stellar properties influence atmospheric escape and the evolution of close-in exoplanets. The case of the warm Neptune GJ 436b, which revealed extremely deep transit signatures in the UV (Kulow et al. 2014; Ehrenreich et al. 2015; Lavie et al. 2017), suggests that members of this class of exoplanets are ideal targets to be characterized through their upper atmosphere. The mild irradiation of these warm Neptunes, which are frequently found around late-type stars, is sufficient to lead to their evaporation, but the combination of stellar wind interactions with the low radiation pressure and low photoionization allows their neutral hydrogen exosphere to extend up to tens of planetary radii (Bourrier et al. 2015b, 2016; Lavie et al. 2017). GJ 436b has a similar radius as the warm Neptune GJ 3470b, but its density is twice as low. Both planets orbit at similar distance (in stellar radii) from $\mathrm{M}$ dwarfs, but GJ3470b is hosted by an earlier-type star. Together, these systems thus offer the possibility of investigating how stellar irradiation and planetary properties affect the upper atmosphere and evolution of warm Neptunes.

Following up on the study of GJ 436b, we obtained Lyman- $\alpha$ observations of GJ3470b as part of the HST Panchromatic Comparative Exoplanet Treasury program (PanCET, GO 14767, P.I. Sing \& Lopez-Morales), which targets 20 exoplanets for the first large-scale simultaneous UltraViolet, Optical, Infrared (UVOIR) comparative study of exoplanets. GJ 3470b was discovered through radial velocity and transit observations by Bonfils et al. (2012). It has a low bulk density $\left(0.8 \mathrm{~g} \mathrm{~cm}^{-3}\right.$; Awiphan et al. 2016) that is indicative of a hydrogen-helium-rich atmosphere (Demory et al. 2013; Chen et al. 2017). With a period of $~ 3.3$ days and a moderately bright and nearby M1.5 dwarf host star $(V=12.3, d=29.5 \mathrm{pc})$, GJ $3470 \mathrm{~b}$ is a favorable target for atmospheric characterization through transit spectroscopy. Its near-infrared transmission spectrum shows no atmospheric features at the precision obtainable with HST/WFC3 in staring mode (Ehrenreich et al. 2014) or from the ground (Crossfield et al. 2013). In the optical, several studies point to a tentative increase in the planet radius toward short wavelengths (Fukui et al. 2013; Nascimbeni et al. 2013; Biddle et al. 2014; Dragomir et al. 2015; Awiphan et al. 2016; Chen et al. 2017). As in HD 189733b, HD 209458b, WASP-6b, and HAT-P-12b (Lecavelier Des Etangs et al. 2008a,c; Nikolov et al. 2015; Sing et al. 2016), this slope could be attributed to Rayleigh scattering in an extended atmosphere of molecular hydrogen and helium. The low gravitational potential of GJ 3470b and the strong highenergy irradiation from its host star suggests that it has an extended upper atmosphere (Salz et al. 2016). Here we report on the first transit observations of GJ 3470b in the H I Lyman- $\alpha$ line, aimed at detecting its extended exosphere of neutral hydrogen. We present the observations in Sect. 2 and describe their analysis in Sect. 3. We derive from our observations the intrinsic Lyman- $\alpha$ line of GJ 3470 in Sect. 4 and its XUV spectrum in Sect. 5. The reconstructed stellar spectrum is used in Sect. 6.1 to interpret the Lyman- $\alpha$ transit observations of GJ 3470b with our $3 \mathrm{D}$ numerical model of evaporating atmosphere. We discuss our results in Sect. 7.

\section{Observations and data reduction}

\subsection{HST STIS observations}

We observed the M dwarf GJ 3470 with the Space Telescope Imaging Spectrograph (STIS) instrument on board the Hubble Space Telescope (HST). We used the STIS/G140M grating (spectral range 1195-1248 , spectral resolution $\sim 20 \mathrm{~km} \mathrm{~s}^{-1}$ ) to measure the transit of GJ 3470b in the stellar Lyman- $\alpha$ line. Three visits of five consecutive HST orbits each were scheduled on 28 November 2017 (Visit A), 4 December 2017 (Visit B), and 7 January 2018 (Visit C). Visits were centered around the planet mid-transit to provide sufficient coverage before and after the transit, and were scheduled at slightly different orbital phases to increase the temporal sampling (see, e.g., Fig. 4). Scientific exposures lasted $2154 \mathrm{~s}$ in all HST orbits, except during the first orbit, which was shorter because of target acquisition (exposure time $1824 \mathrm{~s}$ ). Data obtained in time-tagged mode were reduced with the CALSTIS pipeline, which includes the flux and wavelength calibration, and were divided in all HST orbits into six sub-exposures with duration 304 or $359 \mathrm{~s}$.

Geocoronal airglow emission from the upper atmosphere of Earth (Vidal-Madjar et al. 2003) contaminates the stellar Lyman- $\alpha$ emission line in the raw data (Fig. 2). Although 
Table 1. Physical properties of the GJ 3470 system.

\begin{tabular}{llcc}
\hline \hline Parameters & Symbol & Value & Reference \\
\hline Distance from Earth & $D_{*}$ & $29.45 \pm 0.07 \mathrm{pc}$ & \\
Star radius & $R_{*}$ & $0.547 \pm 0.018 R_{\odot}$ & Awiphan et al. 2016 \\
Star mass & $M_{*}$ & $0.539_{-0.043}^{+0.047} M_{\odot}$ & Demory et al. 2013 \\
Star rotation period & $P_{*}$ & $20.70 \pm 0.15 \mathrm{~d}$ & Biddle et al. 2014 \\
Star age & $\tau_{*}$ & $\sim 2 \mathrm{Gyr}$ & This work \\
Heliocentric stellar radial velocity & $\gamma_{* / \odot}$ & $26.51691 \pm 5.3 \times 10^{-4} \mathrm{~km} \mathrm{~s}^{-1}$ & Bonfils et al. 2012 \\
Planet radius (358 nm) & $R_{\mathrm{p}}$ & $4.8 \pm 0.2 R_{\text {Earth }}$ & Chen et al. 2017 \\
Planet mass & $M_{\mathrm{p}}$ & $13.9 \pm 1.5 M_{\text {Earth }}$ & Demory et al. 2013 \\
Orbital period & $P_{\mathrm{p}}$ & $3.33665173 \pm 5.9 \times 10^{-7}$ days & Chen et al. 2017 \\
Transit center & $T_{0}$ & $2455983.70417 \pm 0.00011 \mathrm{BJD}$ & Chen et al. 2017 \\
Semi-major axis & $a_{\mathrm{p}}$ & $0.0355 \pm 0.0019 \mathrm{au}$ & Awiphan et al. 2016 \\
Eccentricity & $e$ & $0.086_{-0.036}^{+0.050}$ & \\
Argument of periastron & $\omega$ & $-123.5_{-44.4}^{+21.9^{\circ}}$ & \\
Inclination & $i_{\mathrm{p}}$ & $89.13_{-0.34}^{+0.26^{\circ}}$ & Awiphan et al. 2016 \\
\hline
\end{tabular}

Notes. The distance from GJ 3470 was derived from its Gaia parallax measurement (33.96 \pm 0.06 mas; Gaia Collaboration 2016, 2018). The values for $e$ and $\omega$ were derived from radial velocity measurements of GJ 3470 (X. Bonfils, priv. comm.).

CALSTIS corrects the final 1D spectra for airglow contamination, it is recommended to treat the regions where the airglow is stronger than the stellar flux with caution. In these regions, the airglow-corrected flux is usually overestimated (Fig. 2). The strength and position of the airglow varies with the epoch of observation, affecting different parts of the spectrum. Therefore, we excluded from our analysis the wavelength window 1215.70-1215.86 $\AA$ in Visit A, 1215.70-1215.81 $\mathrm{A}$ in Visit B, and 1215.61-1215.78 $\AA$ in Visit C, defined in the heliocentric rest frame.

\subsection{Flux calibration}

STIS observations are affected by variations in the telescope throughput caused by thermal variations that the HST experiences during each orbit (e.g., Brown et al. 2001; Sing et al. 2008; Huitson et al. 2012). This "breathing" effect modifies the flux balance within an HST orbit, and is detected in the three visits (Fig. 3). As expected from previous measurements with the G140M grating, the shape and amplitude of the breathing variations change between visits, but the orbit-to-orbit variations within a single visit are both stable and highly repeatable (e.g., Bourrier et al. 2013; Ehrenreich et al. 2015). Correcting for the breathing variations is particularly important with respect to the first-orbit exposure because it is shorter and shifted to later HST orbital phases than subsequent exposures (Fig. 3). As a result, the average flux over the first orbit is subjected to a different bias than the other orbits.

We fit a breathing model based on Bourrier et al. (2017b) to the sub-exposure spectra integrated over the entire Lyman- $\alpha$ line (1214.7-1216.7 $\AA$ minus the range contaminated by the airglow). This choice was motivated by the achromaticity of the breathing variations, and by the need for a high signal-to-noise ratio $(\mathrm{S} / \mathrm{N})$ to ensure an accurate correction. The breathing was modeled as a polynomial function phased with the period of the HST around the Earth $\left(P_{\mathrm{HST}}=96 \mathrm{~min}\right)$. The nominal flux unaffected by the breathing effect was fit for in each HST orbit, to prevent the overcorrection of putative orbit-to-orbit variations caused by the star or the planet. The model was oversampled in time and averaged

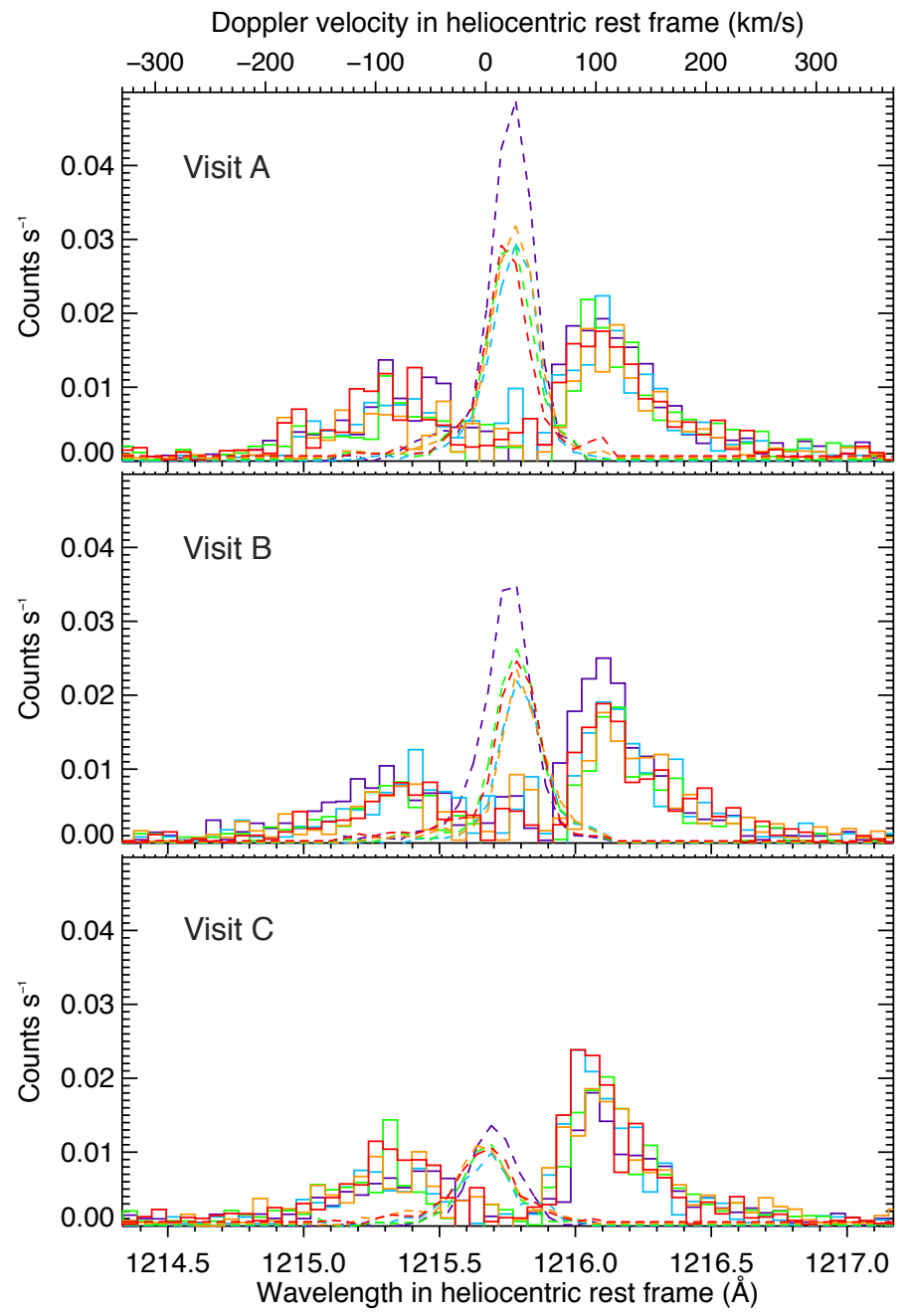

Fig. 2. Raw spectra of GJ 3470 Lyman- $\alpha$ line (histograms) after correction for the geocoronal emission line (superimposed as a dashed line). Colors correspond to HST consecutive orbits in each visit (orbits 1-5 are plotted in purple, blue, green, orange, and red). 

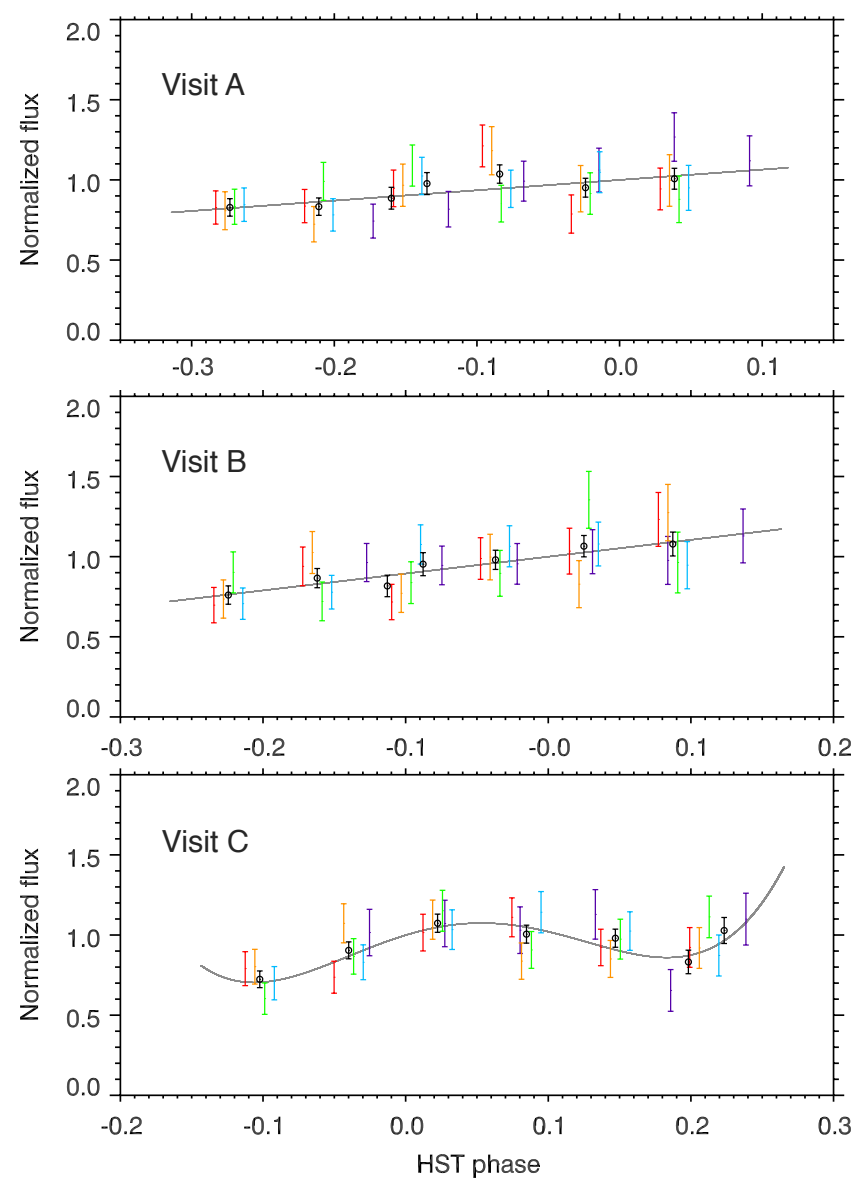

Fig. 3. Lyman- $\alpha$ fluxes for sub-exposures integrated over the entire line and phase-folded on the HST orbital period $\left(P_{\mathrm{HST}}=96 \mathrm{~min}\right)$. The phase is between -0.5 and 0.5 . Solid gray lines correspond to the best-fit breathing model to the data, and were scaled to the same nominal flux for the sake of comparison. Black points show sub-exposures binned manually to highlight the breathing trend. Colors correspond to HST consecutive orbits in each visit (orbits $1-5$ are plotted in purple, blue, green, orange, and red).

within the time window of each exposure before comparison with the data. We used the Bayesian information criterion (BIC; Liddle 2007) as a merit function to determine the best polynomial degree for the breathing variations. We obtained linear variations in Visits $\mathrm{A}$ and $\mathrm{B}$, and a fourth-order polynomial function in Visit C (Fig. 3). The spectra in each sub-exposure were then corrected by the value of the best-fit breathing function at the time of mid-exposure.

\section{Transit analysis in the Lyman- $\alpha$ line}

We searched for flux variations between the Lyman- $\alpha$ spectra of GJ 3470 that would arise from planetary absorption or stellar variability, following the same approach as in previous studies (e.g., Bourrier et al. 2013, 2017b). Spectra were first compared two by two in each visit to identify those showing no significant variations, which could be considered as representative of the quiescent, unocculted stellar line. This was done by searching for all features characterized by flux variations with an $\mathrm{S} / \mathrm{N}$ higher than 3 , and extending over more than 3 pixels $(\sim 0.16 \AA$, higher than STIS spectral resolution). Then, we coadded these stable spectra to create a reference for the intrinsic stellar line in each visit, which was used to characterize the variable features observed in the other spectra. We caution that noise in the spectra makes it difficult to precisely identify the spectral range of a given variable feature. Nonetheless, signatures consistent with absorption of the quiescent stellar line were identified in each visit. We detail their analysis in the following sections, and we plot in Fig. 4 the transit light curves in the spectral bands that showed absorption or bands that remained stable over each visit.

\subsection{Visit $A$}

Lyman- $\alpha$ line spectra are consistent between observations taken outside of the transit (orbits 1,2, and 5). Compared to their average, taken as reference for the intrinsic stellar line, orbits 3 and 4 both revealed absorption signatures in the blue and red wings of the line. These orbits were taken near the ingress and egress of the planetary transit, respectively.

- Red wing: the signatures are detected within a similar spectral range in orbits 3 and $4\left(+21\right.$ to $\left.+60 \mathrm{~km} \mathrm{~s}^{-1}\right)$, with absorption depths of $53.8 \pm 13.0 \%$ and $44.7 \pm 14.1 \%$, respectively (Fig. 4).

- Blue wing: the most significant absorption signatures are found at high velocities, within -163 to $-124 \mathrm{~km} \mathrm{~s}^{-1}$ $(46.7 \pm 14.5 \%)$ in orbit 3 , and within -150 to $-71 \mathrm{~km} \mathrm{~s}^{-1}$ $(43.4 \pm 9.1 \%)$ in orbit 4 . However, a visual inspection and the analysis of an average spectrum of orbits 3 and 4 suggest that absorption extends to lower velocities. In the range -150 to $-32 \mathrm{~km} \mathrm{~s}^{-1}$, we measure consistent absorption depths of $28.8 \pm 9.7 \%$ and $32.8 \pm 9.5 \%$ for orbits 3 and 4 , respectively (Fig. 4).

\subsection{Visit $B$}

Lyman- $\alpha$ line spectra are also consistent between out-of-transit orbits 1-2-5 in Visit B, except for a significantly higher flux at the peak of the red wing in orbit 1 (between +61 and $+126 \mathrm{~km} \mathrm{~s}^{-1}$ ). After scaling spectra from Visits A and B to the same flux level, we found that Visit B spectra in orbits 2 and 5 are in good agreement with the reference spectrum from Visit A. This suggests that the feature in the first orbit of Visit B is a transient brightening of the quiescent stellar line. We thus built the reference spectrum for Visit B as the average between orbits 1 (excluding the brightened region), 2, and 5. Compared to this reference, we identified absorption signatures in orbits 3 and 4, obtained during the planetary transit and shortly after the egress (Fig. 4).

- Red wing: the strongest signature is detected in orbit 3 $\left(38.9 \pm 11.5 \%\right.$ within +8 to $\left.+74 \mathrm{~km} \mathrm{~s}^{-1}\right)$, while orbit 4 shows a marginal signature at higher velocities $(26.7 \pm 9.2 \%$ within +34 to $+87 \mathrm{~km} \mathrm{~s}^{-1}$ ).

- Blue wing: the most significant absorption signatures are found within -110 to $-18 \mathrm{~km} \mathrm{~s}^{-1}(53.3 \pm 14.2 \%)$ in orbit 3, and within -97 to $-57 \mathrm{~km} \mathrm{~s}^{-1}(61.9 \pm 13.4 \%)$ in orbit 4 .

\subsection{Visit $C$}

Visit $\mathrm{C}$ revealed short-term variability that makes the identification of quiescent or variable spectra more difficult. The largest variation occurs in the first orbit at the peak of the red wing $\left(+38\right.$ to $\left.+116 \mathrm{~km} \mathrm{~s}^{-1}\right)$, with the Lyman- $\alpha$ line distorted and significantly fainter than in subsequent orbits. We also observe brightenings in the far red wing in orbit 4, and at the peak of the blue wing in orbit 5 . However, these latter variations are marginal, and we thus built the reference spectrum for Visit $\mathrm{C}$ as the average between orbits 1 (excluding the fainter region), 2, and 5 . These orbits were obtained well outside of the 


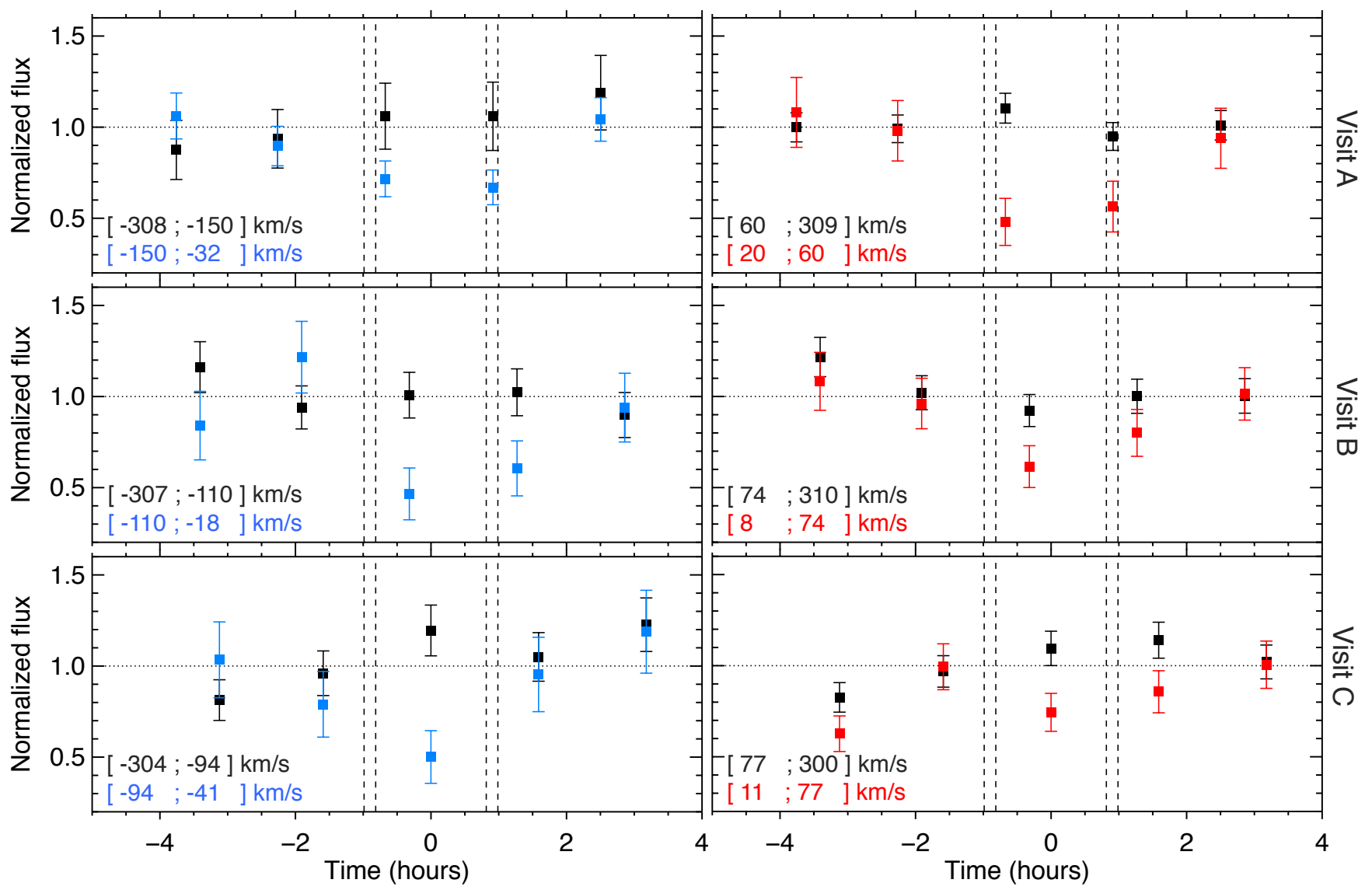

Fig. 4. Lyman- $\alpha$ line flux integrated over bands of interest in Visits A, B, and C as a function of time relative to the mid-transit of GJ $3470 \mathrm{~b}$ (contacts are shown as vertical dashed lines). The spectral ranges of the bands are indicated in each sub-panel. For all visits, blue and red points correspond to bands showing absorption in the blue and the red wing, respectively. We note that the bands were optimized for all orbits in a visit, and do not necessarily show the strongest absorption in a given orbit. Black points correspond to the complementary of the absorbed regions up to the limit of the wings. Visits A and B show stable flux levels in the wings, except for a flux spike at the peak of the red wing in Visit B orbit 1, while Visit $\mathrm{C}$ displays short-term variability. Fluxes are normalized by the average flux over orbits 1, 2, and 5 (excluding spurious ranges in the red wing for Visits B and C, see text).

planetary transit, close to their counterparts that we used as reference in Visits A and B (Fig. 4). Compared to the reference spectrum of Visit $\mathrm{C}$, we identified absorption signatures in orbits 3 and 4, obtained at mid-transit and after the egress.

- Red wing: absorption signatures in the red wing are marginal, but interestingly, they overlap with the signatures detected in previous visits. The most significant signatures are found within +38 to $+77 \mathrm{~km} \mathrm{~s}^{-1}(56.7 \pm 14.7 \%)$ in orbit 3 , and within +51 to $+90 \mathrm{~km} \mathrm{~s}^{-1}(25.9 \pm 8.9 \%)$ in orbit 4.

- Blue wing: orbit 3 shows significant absorption between -94 and $-41 \mathrm{~km} \mathrm{~s}^{-1}(56.7 \pm 14.7 \%)$, whereas we do not detect any significant absorption signature in orbit 4 .

\subsection{Summary}

The stellar Lyman- $\alpha$ line profile of GJ 3470 remains mostly stable within each visit (but see Sect. 4 across the visits). Absorption signatures are detected in both wings of the Lyman- $\alpha$ line in all visits. They are located in similar spectral ranges between about -150 and $+90 \mathrm{~km} \mathrm{~s}^{-1}$ at maximum, although absorption beyond $-100 \mathrm{~km} \mathrm{~s}^{-1}$ is only measured in Visit A. The core of GJ 3470 Lyman- $\alpha$ line cannot be analyzed because it is fully absorbed by the interstellar medium (ISM). Nonetheless, we measure absorption down to the airglow boundaries (varying between -40 and $+20 \mathrm{~km} \mathrm{~s}^{-1}$ due to the differences in airglow position and width between visits), which suggests that it extends to lower absolute velocities. This would be expected if the planetary atmosphere is the source for the absorbing neutral hydrogen atoms, a large portion of which would move with low radial velocities near the transit when they are still in the vicinity of the planet. The light curves plotted over the absorbed spectral ranges in Fig. 5 show that the Lyman- $\alpha$ line transit keeps a similar shape over the three visits (absorption depths are reported in Table 2). Except for spurious variations in the first orbits of Visits B and C, which are likely stellar in origin, spectra obtained before $-1.5 \mathrm{~h}$ and after $2 \mathrm{~h}$ show no variations in any of the visits, indicating the absence of neutral hydrogen occulting the star long before or after the transit. In contrast, absorption is consistently detected during and shortly after the planetary transit (Fig. 4).

The repeatability of the absorption signatures, their spectral ranges spread around the planet radial velocity $\left(\sim 5 \mathrm{~km} \mathrm{~s}^{-1}\right.$ at mid-transit), and their phasing with the planetary transit allow us to conclude the detection of an extended upper atmosphere of neutral hydrogen around the warm-Neptune GJ 3470b. We plot in Fig. 6 the absorbed and stable spectra averaged over the three visits to highlight the signature from this extended atmosphere. We measure absorption depths of $35 \pm 7 \%$ in the blue wing, between -94 and $-41 \mathrm{~km} \mathrm{~s}^{-1}$, and $23 \pm 5 \%$ in the red wing, between 23 and $76 \mathrm{~km} \mathrm{~s}^{-1}$ (Table 2). 


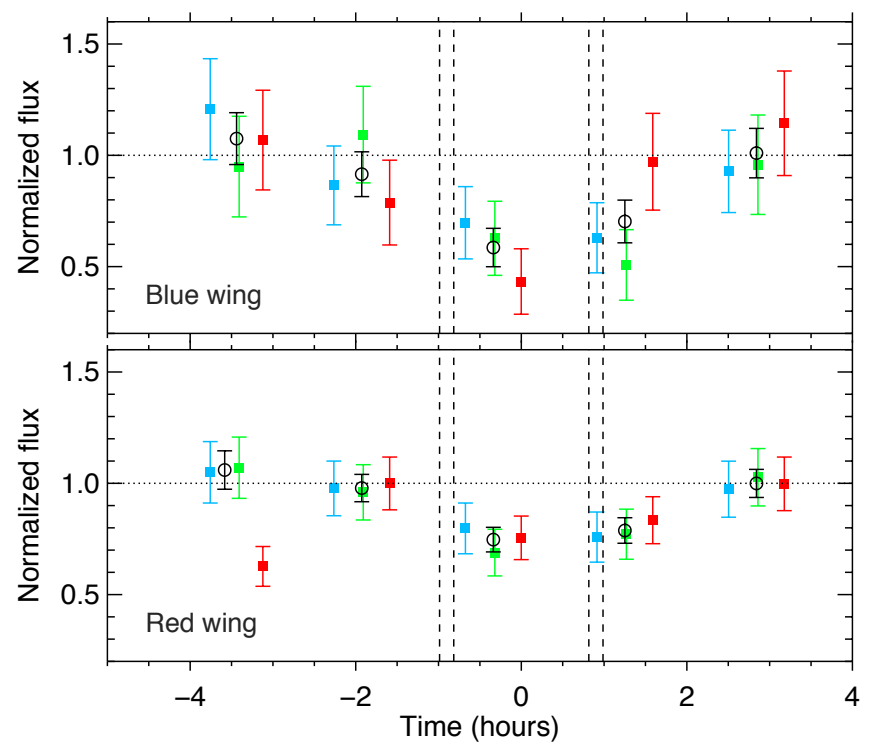

Fig. 5. Flux integrated in the blue wing of the Lyman- $\alpha$ line over -94 to $-41 \mathrm{~km} \mathrm{~s}^{-1}$ (upper panel), and in the red wing over $23-76 \mathrm{~km} \mathrm{~s}^{-1}$ (lower panel). Visits A, B, and C are plotted in blue, green, and red. Fluxes are normalized by the average flux over orbits 1,2, and 5. Time is relative to the mid-transit of GJ $3470 \mathrm{~b}$ (contacts are shown as vertical dashed lines). Exposures occurring at similar times in each visit have been binned into the black points. Orbit 1 in Visit $\mathrm{C}$ has been excluded from the normalization, and the binning because of its spurious flux decreases between +38 and $+116 \mathrm{~km} \mathrm{~s}^{-1}$.

Table 2. Absorption depths measured in the blue $\left(-94\right.$ to $\left.-41 \mathrm{~km} \mathrm{~s}^{-1}\right)$ and red wings $\left(23-76 \mathrm{~km} \mathrm{~s}^{-1}\right)$ of the Lyman- $\alpha$ line.

\begin{tabular}{lcccc}
\hline \hline & \multicolumn{2}{c}{ Blue wing } & \multicolumn{2}{c}{ Red wing } \\
& In-transit & Egress & In-transit & Egress \\
\hline Visit A & $30.7 \pm 16.3$ & $36.5 \pm 15.9$ & $19.0 \pm 11.2$ & $24.4 \pm 10.9$ \\
Visit B & $37.8 \pm 16.7$ & $49.1 \pm 16.0$ & $29.5 \pm 10.3$ & $23.6 \pm 10.9$ \\
Visit C & $56.9 \pm 14.8$ & $2.5 \pm 22.0$ & $24.0 \pm 9.6$ & $17.2 \pm 10.2$ \\
\hline All visits & $41.8 \pm 8.7$ & $29.4 \pm 9.7$ & $24.2 \pm 5.4$ & $21.8 \pm 5.5$ \\
\hline
\end{tabular}

\section{Lyman- $\alpha$ line of GJ 3470}

\subsection{Reconstruction of the intrinsic line profiles}

The profile of the intrinsic stellar Lyman- $\alpha$ line, before absorption by the ISM, is required to calculate the radiation pressure acting on escaping neutral hydrogen atoms, and can be used to estimate the stellar UV emission (see Sect. 5). We used the outof-transit spectra taken as reference to reconstruct the intrinsic stellar Lyman- $\alpha$ line in each visit. To use as much information as possible, we included the stable wings of the in-transit spectra where no absorption was detected. We followed the same procedure as in previous studies (e.g., Bourrier et al. 2015a, 2017b): a model profile of the intrinsic stellar line is absorbed by hydrogen and deuterium in the ISM, convolved with STIS line spread function (LSF), and fit to the data. The model is oversampled in wavelength, and rebinned over the STIS spectral table after convolution.

We found that a single-peaked Voigt profile provides the best fit to the intrinsic Lyman- $\alpha$ line, compared to other typical line profiles with two peaks or Gaussian components (e.g., Wood et al. 2005). This strengthens the trend noted in Bourrier et al. (2017a) that M dwarfs show no self-reversal in

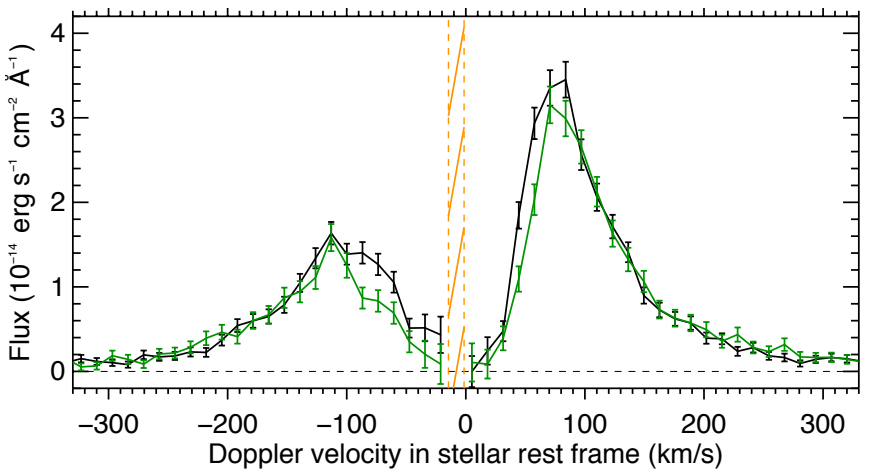

Fig. 6. Average Lyman- $\alpha$ line spectra of GJ 3470b over the three visits, during the transit of the extended neutral hydrogen atmosphere (green spectrum) and outside (black spectrum). The dashed orange region is contaminated by the airglow in all visits.

their core, while $\mathrm{K}$ dwarfs sometimes show double-peaked profiles and G-type stars always do. K dwarfs might thus have transition regions, where the core of the Lyman- $\alpha$ line is formed (Vernazza et al. 1981), that have intermediate structures in between those of G-type stars and M dwarfs. We fit the spectra from all visits together using the Markov chain Monte Carlo (MCMC) Python software package emcee (Foreman-Mackey et al. 2013). The theoretical intrinsic line profile is specific to each visit and is defined by its centroid $\gamma_{\mathrm{Ly}-\alpha}$, its temperature $T_{\mathrm{Ly}-\alpha}$ (assuming pure thermal broadening), its damping parameter $a_{\mathrm{damp}}$, and its total flux $F_{\mathrm{Ly}-\alpha}(1 \mathrm{au})$. The absorption profile of the ISM along the line of sight is common to all visits, and defined by its column density of neutral hydrogen $\log _{10} N_{\text {ISM }}(\mathrm{H} \mathrm{I})$, its Doppler broadening parameter $b_{\text {ISM }}(\mathrm{H} \mathrm{I})$, and

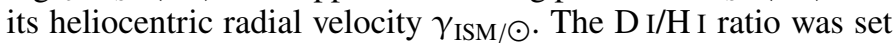
to $1.5 \times 10^{-5}$ (e.g., Hébrard \& Moos 2003; Linsky et al. 2006). Best-fit values for the model parameters are given in Table 3, yielding a good $\chi^{2}$ of 95 for 113 degrees of freedom (128 datapoints and 15 free parameters; Fig. 7).

\subsection{Results and interpretation}

The LISM kinematic calculator ${ }^{1}$, a dynamical model of the local ISM (Redfield \& Linsky 2008), predicts that the line of sight (LOS) toward GJ 3470 crosses the LIC and Gem cloud with heliocentric radial velocities of 18.3 and $33.4 \mathrm{~km} \mathrm{~s}^{-1}$, respectively. The velocity of the ISM cloud we fit is in good agreement with that of the Local Interstellar Cloud (LIC), and its column density $\left(\log _{10} N_{\mathrm{ISM}}(\mathrm{H} \mathrm{I})=18.07 \pm 0.09 \mathrm{~cm}^{-2}\right)$ is consistent with the range of values expected for a star at a distance of $29.5 \mathrm{pc}$ (Fig. 14 in Wood et al. 2005).

The total flux in the observed and reconstructed Lyman- $\alpha$ lines show no significant variations between the three visits (Table 3). The best-fit model for the line averaged over the three visits is shown in Fig. 7. It yields a total flux $F_{\mathrm{Ly}-\alpha}(1 \mathrm{au})=$ $3.64 \pm 0.15 \mathrm{erg} \mathrm{cm}^{-2} \mathrm{~s}^{-1}$, which is in remarkable agreement with the value of $3.7 \mathrm{erg} \mathrm{cm}^{-2} \mathrm{~s}^{-1}$ derived from the empirical relation in Youngblood et al. (2016) and the rotation period of GJ 3470 (Table 1). We note that uncertainties in the Lyman- $\alpha$ line reconstruction do not affect the measurement of the GJ $3470 \mathrm{~b}$ absorption signature, which is a relative measure between inand out-of-transit spectra both absorbed in the same way by the interstellar medium.

\footnotetext{
1 http://sredfield.web.wesleyan.edu/
} 


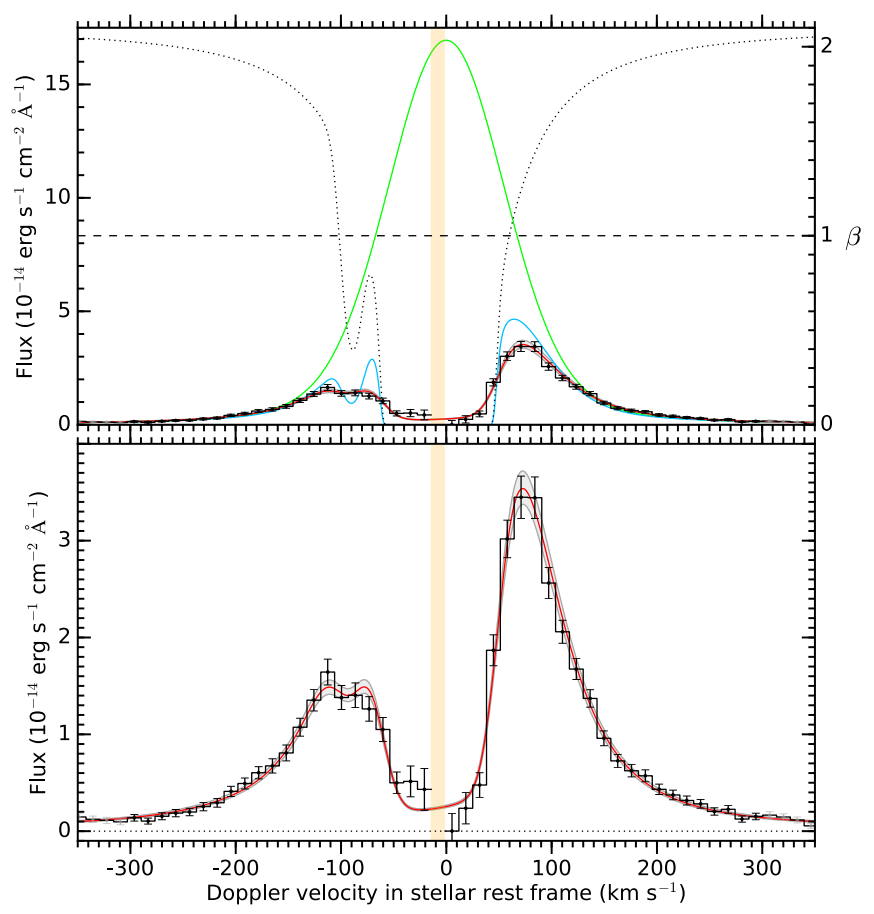

Fig. 7. Lyman- $\alpha$ line profile of GJ 3470 averaged over the three visits. The black histogram shows the observed spectra, fit over points with black error bars. The orange band shows the regions that are too contaminated by geocoronal emission to be included in the fit. The green line is the best fit for the intrinsic stellar line profile. It yields the blue profile after absorption by the interstellar medium, whose profile is plotted as a dotted black line (ISM absorption in the range $0-1$ has been scaled to the vertical axis range). The red line shows the line profile fit to the data, after convolution with the STIS LSF. Gray bands delimitate the $1 \sigma$ envelopes of the best-fit profiles. The theoretical intrinsic stellar line profile also corresponds to the profile of the ratio $\beta$ between radiation pressure and stellar gravity, which is reported on the right axis in the top panel. Bottom panel: zoom of the observed Lyman- $\alpha$ line and its best-fit model.

Our reconstruction for the intrinsic Lyman- $\alpha$ line of GJ 3470 shows that radiation pressure is strong enough for neutral hydrogen atoms escaping from the planet to be accelerated away from the star. Radiation pressure from this M1.5 dwarf indeed overcomes its gravity by up to a factor 2 (Fig. 7), which is intermediate between the factor 3 associated with the K0 dwarfs HD 189733 and HD 97658 (Bourrier \& Lecavelier des Etangs 2013; Bourrier et al. 2017b) and the factor 0.7 associated with the M2.5 dwarf GJ 436 (Bourrier et al. 2015a). We thus expect the putative hydrogen exosphere of GJ $3470 \mathrm{~b}$ to be in an intermediate regime of radiative blow-out, in between the stronger radiative blow-out acting on the comet-like exospheres of HD 209458 (Lecavelier Des Etangs et al. 2008b) or HD 189733b (Bourrier \& Lecavelier des Etangs 2013), and the radiative braking shaping the giant exosphere of GJ 436b (Bourrier et al. 2015a, 2016).

\section{XUV irradiation of the upper atmosphere}

In addition to the Lyman- $\alpha$ line (1215.67 $\AA$ ), we identified the stellar emission lines of Si III (1206.5 $\left.\AA, \log T_{\max }(\mathrm{K})=4.8\right)$, and the N V doublet $\left(1242.8\right.$ and $\left.1238.8 \AA, \log T_{\max }(\mathrm{K})=5.3\right)$ in the STIS spectra of GJ 3470. These lines are useful tracers of the stellar transition region. To measure their strengths, we averaged the spectra in each visit and fit Gaussian models to the three emission lines following the same approach as in Sect. 4. We assumed that there is no turbulent broadening and that the lines share a common Doppler shift. We allowed for a different continuum level in the range of the Si III line because we found it was overcorrected by CALSTIS to negative values. The best-fit properties for the lines are reported in Table 3 and the corresponding models are displayed in Fig. 8. The radial velocity of the Lyman- $\alpha$, Si III, and N V lines are consistent in each visit, but they are systematically blueshifted with respect to the heliocentric radial velocity of GJ 3470. This is not the expected behavior for high-energy stellar emission lines, which might show physical redshifts due to the structure of the stellar chromosphere (e.g., Linsky et al. 2012). It is therefore likely that these marginal shifts arise from an offset in STIS wavelength calibration. We accounted for this offset in the fit to the ISM properties and in the interpretation of the observed spectra hereafter.

Most of the stellar EUV spectrum is not observable from Earth because of ISM absorption. We have thus constructed a synthetic XUV spectrum of GJ 3470 in the region 1-1200 based on a coronal model, following Sanz-Forcada et al. (2011). We based our coronal model on the X-ray spectra obtained from the XMM-Newton archive (obs ID 763460201, observed in 2015/04/15, P.I. Salz) for the coronal region, and on the UV fluxes derived for the Si III and NV lines for the transition region (we used their weighted mean since no significant variations were detected between the three visits). The spectral fit gives an X-ray $(0.12-2.48 \mathrm{keV}$, or $5-100 \AA)$ luminosity of $2.3 \times 10^{27} \mathrm{erg} \mathrm{s}^{-1}$. The model-derived luminosities in different EUV and FUV bands are $1.2 \times 10^{28} \mathrm{erg} \mathrm{s}^{-1}(100$ $920 \AA), 5.6 \times 10^{27} \mathrm{erg} \mathrm{s}^{-1}(920-1200 \AA)$ and $2.7 \times 10^{27} \mathrm{erg} \mathrm{s}^{-1}$ (100-504 $\AA$ ). The coronal model also predicts a Lyman- $\alpha$ flux of $7.0 \mathrm{erg} \mathrm{cm}^{-2} \mathrm{~s}^{-1}$ at $1 \mathrm{au}$, that is, a factor of $\sim 2$ only of difference with the flux derived from the observations (Table 3 ). This is remarkable, considering that the coronal model is not expected to correctly predict the Lyman- $\alpha$ flux. Further details about the coronal model will be given in Sanz-Forcada et al. (in prep.). We show in Fig. 9 the comparison between our XUV spectrum and the broadband fluxes obtained with the semi-empirical relations from Linsky et al. (2014) and the intrinsic Lyman- $\alpha$ fluxes derived from our observations. Lyman- $\alpha$ is formed in a limited range of temperature in the transition region, thus the use of a coronal model based on actual data should always be preferred to correctly sample the transition region and coronal temperatures where emission lines in the XUV band are originated (Sanz-Forcada \& Ribas 2015).

The XUV spectrum derived between 5 and $911.8 \AA$ yields a photoionization lifetime of $55 \mathrm{~min}$ for neutral hydrogen atoms at the semi-major axis of GJ 3470 b (details on the calculation can be found in Bourrier et al. 2017b). For comparison, we obtain a lifetime of $2.6 \mathrm{~h}$ when using the EUV flux derived from the Linsky et al. (2014) relations. While other processes like radiation pressure or the strength of the planetary outflow play a role in shaping exospheres, the short photoionization lifetime at GJ 3470b likely prevents escaping hydrogen atoms from remaining neutral as far away from the planet as in the giant exosphere of GJ $436 \mathrm{~b}$ (photoionization lifetime $\sim 12 \mathrm{~h}$ ).

\section{3D simulations of the upper atmosphere of GJ 3470b}

\subsection{Modeling with the EVE code}

We interpreted the Lyman- $\alpha$ transit spectra of GJ 3470b using 3D simulations of its upper atmosphere with the EVaporating Exoplanets (EVE) code (Bourrier \& Lecavelier des Etangs 2013; 


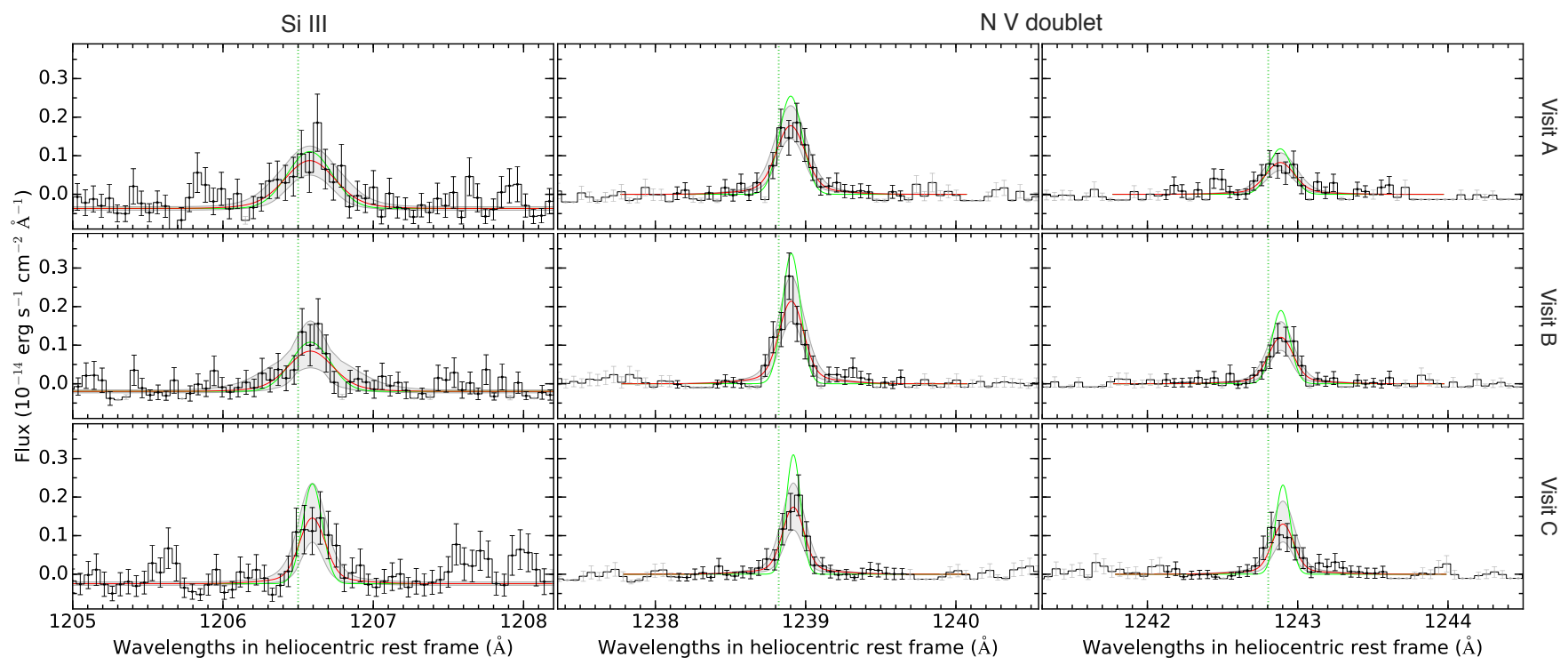

Fig. 8. Spectral profiles of the GJ 3470 Si III line and N V doublet. Each row corresponds to a visit. Black histograms show the observed spectra, fit over points with black error bars. The green lines are the best fits for the intrinsic stellar line profile, which yield the red line after convolution with the STIS LSF. Gray bands delimitate the $1 \sigma$ envelopes of the best-fit profiles. Vertical dashed lines identify the lines transitions in the Sun rest frame.

Table 3. Properties derived from the fit to the GJ 3470 stellar lines.

\begin{tabular}{|c|c|c|c|c|c|c|}
\hline & & Parameter & Visit 1 & Visit 2 & Visit 3 & Unit \\
\hline \multirow[t]{9}{*}{ Stellar lines } & \multirow[t]{2}{*}{ Radial velocity } & $\gamma_{\mathrm{Si} \text { III+N V }}$ & $19.2 \pm 3.4$ & $20.4 \pm 2.6$ & $23.5 \pm 2.7$ & $\mathrm{~km} \mathrm{~s}^{-1}$ \\
\hline & & $\gamma_{\text {Ly- } \alpha}$ & $21.8 \pm 1.7$ & $22.4 \pm 1.6$ & $25.0 \pm 1.8$ & $\mathrm{~km} \mathrm{~s}^{-1}$ \\
\hline & \multirow[t]{4}{*}{ Total flux } & $F_{\mathrm{Si} \text { III }}(1 \mathrm{au})$ & $20.2 \pm 4.1$ & $15.9_{-3.2}^{+4.0}$ & $15.2 \pm 3.5$ & $10^{-3} \mathrm{erg} \mathrm{cm}^{-2} \mathrm{~s}^{-1}$ \\
\hline & & $F_{\mathrm{NV}}^{1238.8}(1 \mathrm{au})$ & $18.0 \pm 2.4$ & $19.0_{-2.8}^{+2.4}$ & $13.6 \pm 2.1$ & $10^{-3} \mathrm{erg} \mathrm{cm}^{-2} \mathrm{~s}^{-1}$ \\
\hline & & $F_{\mathrm{NV}}^{1242.8}(1 \mathrm{au})$ & $8.3 \pm 1.7$ & $10.7 \pm 1.8$ & $10.2_{-1.8}^{+1.7}$ & $10^{-3} \mathrm{erg} \mathrm{cm}^{-2} \mathrm{~s}^{-1}$ \\
\hline & & $F_{\mathrm{Ly}-\alpha}(1 \mathrm{au})$ & $3.4_{-0.5}^{+0.7}$ & $3.5_{-0.5}^{+0.7}$ & $4.2_{-0.7}^{+0.9}$ & 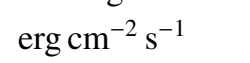 \\
\hline & \multirow[t]{3}{*}{ Temperature } & $T_{\mathrm{Si} \mathrm{III}}$ & $47_{-21}^{+33}$ & $35_{-28}^{+106}$ & $9.0_{-5.1}^{+6.9}$ & $10^{5} \mathrm{~K}$ \\
\hline & & $T_{\mathrm{Nv}}$ & $5.8_{-2.1}^{+2.6}$ & $3.7_{-1.6}^{+2.2}$ & $2.3 \pm 1.6$ & $10^{5} \mathrm{~K}$ \\
\hline & & $T_{\mathrm{Ly}-\alpha}$ & $3.9_{-0.5}^{+0.6}$ & $3.1 \pm 0.4$ & $2.7_{-0.3}^{+0.4}$ & $10^{5} \mathrm{~K}$ \\
\hline \multirow[t]{3}{*}{ ISM } & Radial velocity & $\gamma_{\mathrm{ISM} / \odot}$ & & $18.8_{-1.5}^{+1.4}$ & & $\mathrm{~km} \mathrm{~s}^{-1}$ \\
\hline & Column density & $\log _{10} N_{\mathrm{ISM}}(\mathrm{H} \mathrm{I})$ & & $18.07 \pm 0.09$ & & $\mathrm{~cm}^{-2}$ \\
\hline & Doppler broadening & $b_{\mathrm{ISM}}(\mathrm{HI})$ & & $16.6_{-0.7}^{+0.6}$ & & $\mathrm{~km} \mathrm{~s}^{-1}$ \\
\hline
\end{tabular}

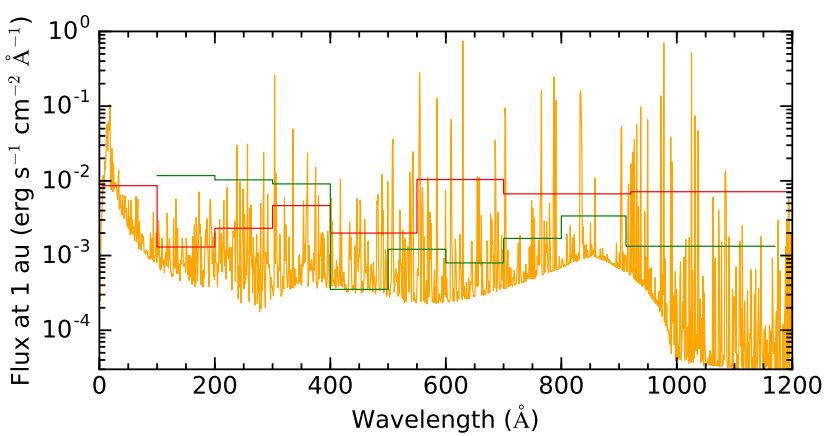

Fig. 9. Synthetic XUV spectrum of GJ 3470 (orange) derived from the coronal model, as explained in the text. The spectrum is integrated over broad spectral bands (red) for comparison with the values derived from the semi-empirical relations in Linsky et al. (2014) using our measured Lyman- $\alpha$ flux (green).
Bourrier et al. 2014, 2015a, 2016). In this code, a planetary system is simulated over time in the star rest frame. A parameterized grid, corresponding to the collisional layers of the atmosphere, is described with a 3D grid in which the density, velocity, and temperature of neutral hydrogen are calculated using analytical functions. The upper boundary of the parameterized atmosphere represents the altitude beyond which species decouple in the collisionless exosphere. Metaparticles of neutral hydrogen, representing groups of atoms with the same properties, are released at this boundary. Their dynamics is calculated using a particle Monte Carlo simulation that accounts for the planet and star gravity, the inertial force linked to the non-Galilean stellar reference frame, radiation pressure, and interactions with the stellar wind (see details in the above papers). The population of neutral hydrogen atoms in the exosphere follows an exponential decay because of stellar photoionization, which is calculated using an input XUV spectrum. Particles in the shadow of the opaque 
planetary disk are not interacting with photons and particles from the star.

At each time step of the simulation, the code calculates the theoretical spectrum seen from Earth as the sum of the local spectra over the discretized stellar disk, accounting for the opaque planetary disk occultation and for the wavelengthdependent absorption from the bottom atmosphere, the exosphere, and the ISM. Atmospheric cells and metaparticles in front of, or behind, the opaque planetary disk do not contribute to the absorption. Theoretical spectra are calculated with a high spectral resolution and temporal cadence compared to the observations. At the end of the simulation, they are downsampled to the resolution of the observed spectra and averaged within the time window of the observed exposures, weighting their relative contribution by the effective overlap with the observed time window. The final spectra, convolved with the instrumental response, are comparable with the observations. Modeling with the EVE code thus allows us to use all information available in an observed dataset, accounting for the partial occultation of the stellar disk during ingress and egress and for 3D effects inherent in the asymetrical structure of extended atmospheres.

\subsection{Application to GJ $3470 b$}

There are two remarkable features in the observations, which we detail below.

(i) Absorption is observed up to high positive velocities ( $\left.\sim 90 \mathrm{~km} \mathrm{~s}^{-1}\right)$ during and shortly after the optical transit. This is challenging to explain by neutral hydrogen gas infalling toward the star. Upper atmospheric layers sheared by the stellar gravity would indeed stream through the L1 Lagrangian point ahead of the planet and occult the star before its transit, not after (e.g., Bourrier et al. 2015a). Interactions between the stellar and planetary magnetic fields might force ionized planetary material to stream toward the star behind the planet, carrying along neutral hydrogen (Matsakos et al. 2015; Strugarek 2016), but radiation pressure or interactions with the stellar wind might stop the infalling flow of neutral hydrogen (e.g., Bisikalo et al. 2013).

Alternatively, the Lorentzian wings from the absorption profile of a dense, extended thermosphere (or damping wings) have been proposed as a possible origin for redshifted Lyman$\alpha$ absorption signatures from exoplanets (Tian et al. 2005; Ben-Jaffel 2008). However, for damping wings to explain the observations of GJ 3470b its thermosphere would have to show a larger extension along the planetary motion than in the perpendicular directions. A spherical thermosphere large enough to yield absorption up to $1.5 \mathrm{~h}$ after the transit would indeed yield a much deeper absorption than observed at mid-transit (see Fig. 5 and next section). To test this scenario, we modified the EVE code so that it can simulate the thermosphere as an ellipsoid with radius $R_{\|}$along the tangent to its orbit, $R_{\perp}^{\text {orb }}$, along the normal to this tangent in the orbital plane, and $R_{\perp}^{\text {norm }}$ along the perpendicular to the orbital plane. The ellipsoid can be off-centered from the planet along the tangent to its orbit by $d_{\|}$(positive when the ellipsoid center is ahead of the planet). Because we only probe column densities along the LOS, we did not try to fit possible inclination of the ellipsoidal atmosphere with respect to the orbital trajectory. We assumed a simple isotropic, hydrostatic density profile, defined by a mean temperature $T_{\text {th }}$ (in K) and the density of neutral hydrogen $n_{\mathrm{th}}$ at $1 R_{\mathrm{p}}$ (in atoms $/ \mathrm{cm}^{3}$ ). We assumed a solar-like composition of atomic hydrogen and helium $(\mu=1.2)$. (ii) Absorption is deeper in the blue wing and extends to higher absolute velocities than in the red wing. This spectral asymmetry is even more pronounced in the planet rest frame, since the radial orbital velocity of GJ $3470 \mathrm{~b}$ is positive during the transit. Blueshifted Lyman- $\alpha$ absorption signatures are a telltale sign of neutral hydrogen exospheres around evaporating planets (e.g., Vidal-Madjar et al. 2003; Lecavelier des Etangs et al. 2012; Ehrenreich et al. 2015), and we thus modeled the exosphere of GJ 3470b using particle simulations of the escaping gas (Sect. 6.1). Metaparticles of neutral hydrogen are released uniformely from the surface of the ellipsoidal atmosphere, with an upward velocity of $5 \mathrm{~km} \mathrm{~s}^{-1}$ typical of outflows from low-gravity planets (Salz et al. 2016). Because the orbit of GJ 3470b is eccentric, the mass-loss rate of neutral hydrogen was set to vary as the inverse square of the distance to the star, with the fit value $\dot{M}_{H^{0}}$ (in $\mathrm{g} \mathrm{s}^{-1}$ ) defined at the semi-major axis. The evolution of neutral hydrogen atoms was calculated under radiation pressure and photoionization using the Lyman- $\alpha$ and XUV spectra derived in Sects. 4 and 5.

The observed and simulated time-series spectra were compared over the velocity range -300 to $300 \mathrm{~km} \mathrm{~s}^{-1}$ (in the star rest frame), excluding the airglow-contaminated regions and the spurious regions in the first exposures of Visits B and C (Sect. 3.4). As shown in the following sections, the detected signature is well explained by radiation pressure acceleration with no evidence for interactions with the stellar wind. We thus followed the same approach as in previous studies (e.g., Bourrier \& Lecavelier des Etangs 2013; Bourrier et al. 2014, 2016), which is to fit unknown mechanisms in our simulations only when the known forces are not sufficient to explain the observations. In contrast to radiation pressure, which is derived directly from the observed Lyman- $\alpha$ line, there are only model-derived estimates for the wind properties of exoplanet host stars, and M-dwarf winds in particular are poorly known (Vidotto \& Bourrier 2017). In summary, the free parameters in the model are the 3D dimensions of the ellipsoidal atmosphere $R_{\|}, R_{\perp}^{\text {orb }}, R_{\perp}^{\text {norm }}$ and its offset with respect to the planet center $d_{\|}$, the temperature $T_{\text {th }}$ and density $n_{\text {th }}$ (at $1 R_{\mathrm{p}}$ ) of neutral hydrogen in the upper atmosphere, and its mass-loss rate $\dot{M}_{H^{0}}$ at the exobase. We explored a grid of these parameters, using the $\chi^{2}$ as the merit function to identify a best-fit model and to study the influence of the atmospheric properties.

\subsection{Structure of the extended atmosphere of GJ $3470 \mathrm{~b}$}

Our simulations suggest that the blueshifted and redshifted absorption signatures from GJ 3470 b probe different regions of its upper atmosphere (Fig. 10). The in-transit to posttransit redshift of the absorption signatures measured in the red wing of the Lyman- $\alpha$ line (Sect. 3) is consistent with gas in the upper atmosphere of GJ 3470b moving with its eccentric orbital motion (Table 1; the planet radial velocity increases from $\sim 0$ to $20 \mathrm{~km} \mathrm{~s}^{-1}$ between about -0.7 and $1.6 \mathrm{~h}$ from mid-transit). Because interstellar neutral hydrogen absorbs the stellar flux completely within about -58 to $43 \mathrm{~km} \mathrm{~s}^{-1}$, we cannot access the saturated core from the atmospheric absorption profile, and part of its damping blue wing that is redshifted by the orbital motion. The rest of the damping blue wing contributes little to the observable absorption because it is located in the blue wing of the stellar Lyman- $\alpha$ line, which is made fainter by interstellar deuterium absorption. Conversely, the orbitalmotion redshift moves the damping red wing of the atmospheric 

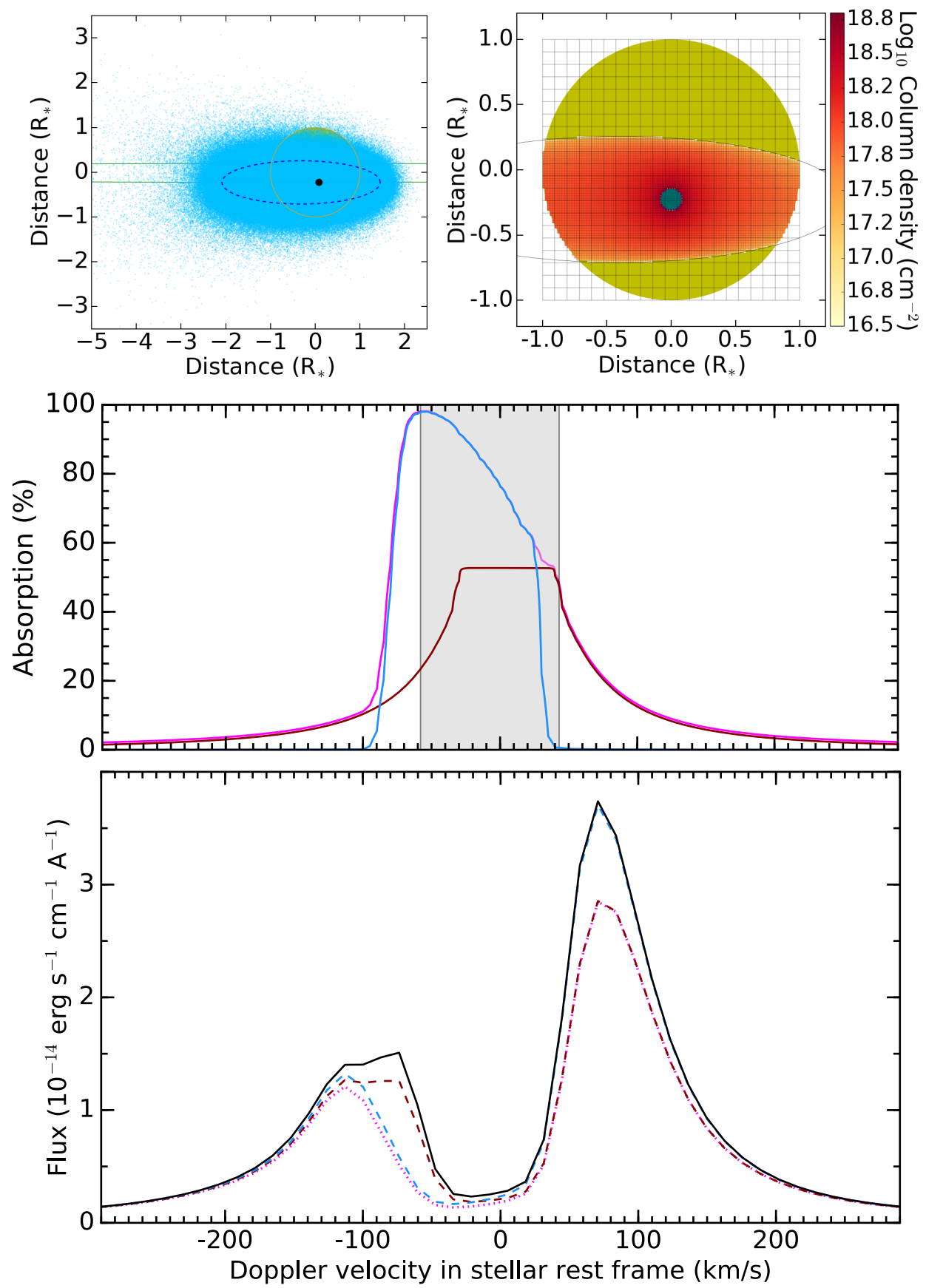

Fig. 10. Contributions to the absorption by the different regions of GJ 3470b upper atmosphere at mid-transit, from one of the best-fit simulations. Top panel: transit view of the exosphere (left panel) and of the ellipsoidal atmosphere (right panel). In the left subpanel we show in projection in the plane of sky the planetary orbit (green curve) and the boundary of the ellipsoidal atmosphere (dashed blue curve). The planet as seen at optical wavelengths is shown as a black and a blue disk in the left and right subpanels, respectively. Middle panel: total absorption profile from GJ 3470 b (magenta), and independent contributions from its exosphere (blue) and ellipsoidal atmosphere (dark red), with neither ISM absorption nor instrumental convolution. Within the shaded region, the stellar flux is entirely absorbed by the ISM. Bottom panel: theoretical Lyman- $\alpha$ line profiles as they would be observed with STIS in Visit C, outside of the transit (black), and during the third orbit at mid-transit with absorption by GJ 3470 b (magenta), its exosphere alone (blue), and its ellipsoidal atmosphere alone (red). There is flux in the full-absorption ISM region because it has been spread by the broad LSF of STIS.

absorption profile into the bright red wing of the observed Lyman- $\alpha$ line, allowing us to measure its signature with a high significance.

Meanwhile, the radiative blow-out of escaping neutral hydrogen atoms combined with the orbital velocity of the planet at the time they escape leads to a blueshifted radial velocity field in the exosphere. As a result, exospheric neutral hydrogen atoms do not contribute to absorption in the observable red wing, and their velocity field is consistent with the spectral range of the absorption measured in the blue wing. Absorption from the exosphere dominates the blueshifted signature during and after the planetary transit.

\subsubsection{Red wing: an elongated upper atmosphere}

Density decreases slowly with altitude in the upper atmospheric layers because of the high temperature. The fit to the red wing signature is thus partly degenerate between the density level, the temperature, and the spatial extension of the ellipsoidal atmosphere. For example, a decrease in temperature of a few thousand Kelvin yields a steeper density profile, which can be compensated for by increasing the overall density of the atmosphere, by increasing its projected surface in the plane of sky, or by increasing its extension along the LOS. Interestingly, however, we found that the spatial extension along the orbital motion could be constrained independently, thanks to the finite duration of the Lyman- $\alpha$ transit in the red wing (Fig. 11). To further investigate the properties of the upper atmosphere of GJ 3470b, we fixed $T_{\text {th }}=7000 \mathrm{~K}$, which is on the order of the temperatures predicted by Salz et al. (2016) for this planet. With this temperature, observations are consistent with densities of neutral hydrogen at $1 R_{\mathrm{p}}$ in between $\sim 10^{8}$ and $10^{10} \mathrm{~cm}^{-3}$, and hereafter, $n_{\text {th }}$ was fixed to $10^{9} \mathrm{~cm}^{-3}$. 

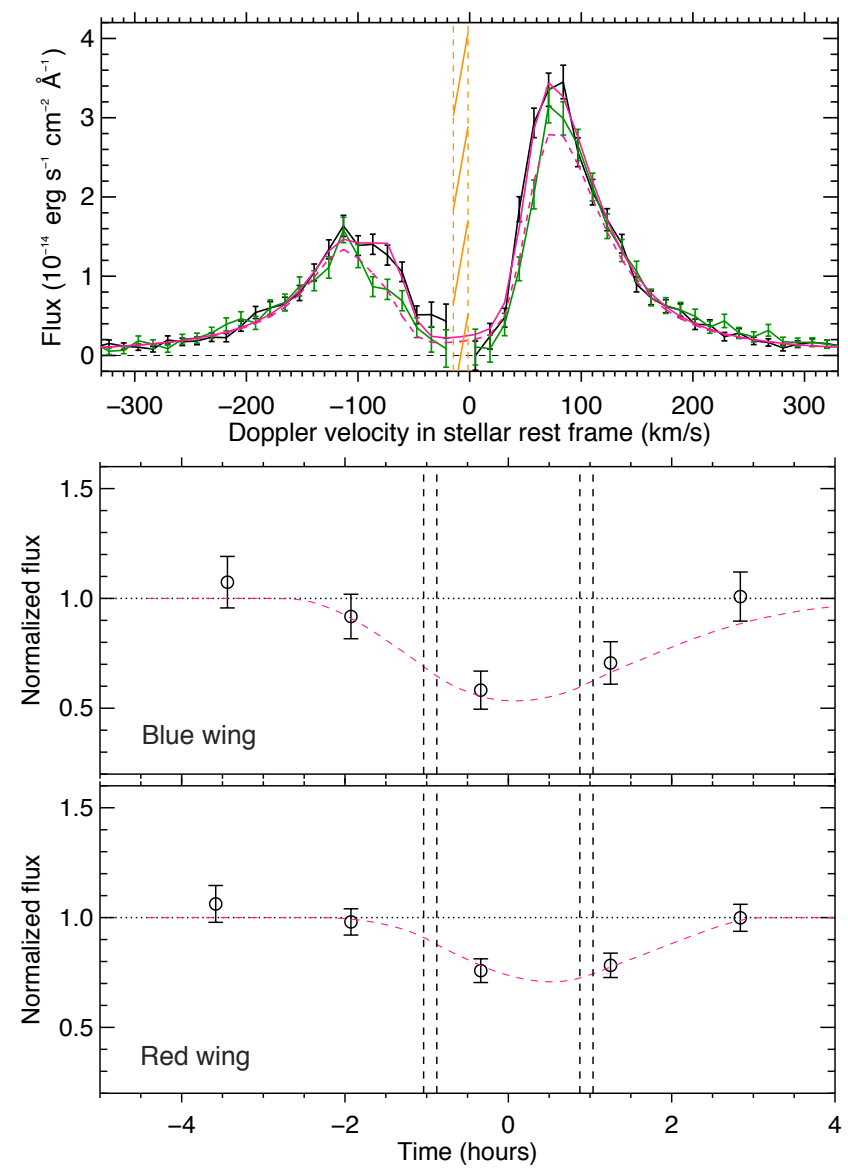

Fig. 11. Best-fit simulation to GJ 3470b Lyman- $\alpha$ spectra (top panel) and corresponding light curves (bottom panels). The solid magenta spectrum, which corresponds to the reconstructed intrinsic stellar spectrum, yields the dashed spectrum and light curves after absorption by the simulated upper atmosphere.

We show in Fig. 10 a best-fit model for the upper atmosphere of GJ 3470b that reproduces the spectral and temporal properties of the absorption signatures in the blue and the red wings well (Fig. 11). It yields a $\chi^{2}$ of 569 for 594 datapoints. The best-fit ellipsoidal atmosphere needs to be offset by $d_{\|}=-4.5 \pm 2.5 R_{\mathrm{p}}$, with a radius $R_{\|}=22_{-3}^{+4} R_{\mathrm{p}}$. These values do not depend on the density and temperature in the atmosphere or on its extension in other dimensions, and suggest that neutral hydrogen gas could be present as far as $\sim 18 R_{\mathrm{p}}$ ahead of the planet, and $\sim 27 R_{\mathrm{p}}$ behind it. The radii of the atmosphere in the plane perpendicular to the orbital motion are correlated with each other because of their similar influence on the column density, and for the chosen atmospheric temperature and reference density, we derive $\mathrm{R}_{\perp}^{\text {orb }}=$ $4_{-2}^{+3} R_{\mathrm{p}}$ and $\mathrm{R}_{\perp}^{\mathrm{norm}}=7_{-2}^{+4} R_{\mathrm{p}}$. The dense envelope of neutral hydrogen surrounding GJ $3470 \mathrm{~b}$ must therefore deviate significantly from a spherical shape to explain the observations. For comparison, the best-fit for a centered, spherical atmosphere yields a $\chi^{2}$ of 580 for a radius of $8 R_{\mathrm{p}}$. The corresponding value for the Akaike information criterion is higher by five points than the fit with an ellipsoidal atmosphere, showing that a spherical atmosphere is less than $10 \%$ as probable to explain the observations.

\subsubsection{Blue wing: a blown-away exosphere}

In the frame of our simulations, Fig. 10 shows that absorption in the blue wing of the Lyman- $\alpha$ line arises from neutral hydrogen atoms that survived stellar photoionization long enough to be accelerated by radiation pressure to high velocities. Because of the strong radiative acceleration (see Sect. 7.3 and Bourrier \& Lecavelier des Etangs 2013), the structure of the outermost exospheric layers is not very sensitive to the altitude and velocity at which the neutral hydrogen atoms originally escaped. The density of high-velocity atoms in these layers, however, depends directly on the mass-loss rate of neutral hydrogen $\dot{M}_{\mathrm{H}^{0}}$. With radiation pressure and photoionization known, $\dot{M}_{\mathrm{H}^{0}}$ is thus tightly constrained by the observations to a value of about $1.5 \times 10^{10} \mathrm{~g} \mathrm{~s}^{-1}$, independent of the other model parameters. We note that even if the exosphere was a mix of planetary atoms and neutralized stellar wind protons (e.g., Kislyakova et al. 2014; Bourrier et al. 2016), each proton would have taken the charge of a planetary atom, and the derived mass-loss rate would still trace atoms that originally escaped from the planet.

We used the XUV spectrum derived between 5 and $911.8 \AA$ (Sect. 5) to estimate the total energy-limited mass-loss rate $\dot{M}^{\text {tot }}$ from the upper atmosphere of GJ 3470b (see, e.g., Watson et al. 1981; Lecavelier des Etangs 2007), accounting for the effects of tides (Erkaev et al. 2007). We obtain $\dot{M}^{\text {tot }}=\eta 8.5 \times 10^{10} \mathrm{~g} \mathrm{~s}^{-1}$, where $\eta$ represents the fraction of the energy input that is available for atmospheric heating. This total mass-loss rate accounts for all escaping elements, including neutral hydrogen, whose loss rate can be defined as $\dot{M}_{H^{0}}=f_{H^{0}} \dot{M}^{t o t}$, where $f_{H^{0}}$ is the fraction of neutral hydrogen in the outflow (see, e.g., Bourrier et al. 2016). Comparing $\dot{M}^{\text {tot }}$ with the value for $\dot{M}_{H^{0}}$ derived from the observations, we obtain $\eta f_{H^{0}} \sim 18 \%$, showing that GJ $3470 \mathrm{~b}$ is subjected to a strong evaporation. Typical theoretical estimations of $\eta$ range between 10 and 30\% (e.g., Lammer et al. 2013; Shematovich et al. 2014; Owen \& Alvarez 2016), suggesting that $f_{H^{0}}$ is larger than $50 \%$.

\section{Discussion}

\subsection{Possible origin for the extended envelope of GJ $3470 b$}

The dimensions derived for the ellipsoidal atmosphere of GJ 3470b are dependent on our assumption of an isotropic density profile. Nonetheless, there must be dense layers of neutral hydrogen gas extending far beyond the gravitational influence of the planet to explain the observations. The Roche lobe of GJ $3470 \mathrm{~b}$ indeed extends up to $5.1 R_{\mathrm{p}}$ along the star-planet axis and has an equivalent volumetric radius of $3.6 R_{\mathrm{p}}$. Even filled with neutral hydrogen and fully opaque, it would yield a maximum absorption of $\sim 8 \%$, much lower than the measured redshifted absorption. While damping wings from an ellipsoidal thermosphere explain this signature well, the atmosphere of an exoplanet cannot continue to expand beyond the Roche lobe without being sheared by stellar gravity and inertial forces. However, our Lyman- $\alpha$ observations only probe column densities of neutral hydrogen, and therefore we cannot constrain the exact position of the gas along the LOS. For example, we would obtain a similar fit to the data if all neutral hydrogen along the LOSs crossing the best-fit ellipsoidal atmosphere was concentrated in a denser shell facing the star, with the same projected surface in the plane of sky. Interestingly, such a structure could arise from the interaction between the stellar and the planetary winds. Using 2D hydrodynamical simulations, Tremblin \& Chiang (2013) simulated the mixing layer formed by the colliding winds. The planetary outflow encounters a bow-shock at altitudes of several planet radii on the starward side, and then forms a thick and dense layer extending over tens of planetari radii along the planet motion. Similarly, 
3D magnetohydrodynamic simulations performed by Bisikalo et al. (2013), Matsakos et al. (2015), or Khodachenko et al. (2017), for instance, show that a strong and dense planetary outflow can move to high altitudes beyond the Roche lobe before intercepting the stellar wind. Part of the shocked, ionized planetary material can then accrete onto the star, while another part is swept back toward the planet and remains confined within its vicinity. Our observations would be sensitive to neutral hydrogen in this confined region, which would be a mix of planetary atoms and stellar wind protons that are neutralized via charge-exchange (Tremblin \& Chiang 2013).

Interestingly, other exoplanets with extended atmospheres of neutral hydrogen have shown tentative redshifted absorption signatures. The evaporating hot Jupiters HD 209458b and HD $189733 \mathrm{~b}$ revealed absorption signatures in between 54$187 \mathrm{~km} \mathrm{~s}^{-1}(5.2 \pm 1.0 \%)$ and $60-110 \mathrm{~km} \mathrm{~s}^{-1}(5.5 \pm 2.7 \%)$, respectively (Vidal-Madjar et al. 2003, 2008; Lecavelier des Etangs et al. 2012; Bourrier et al. 2013). These signatures occurred during the planetary transits, and damping wings from an extended thermosphere have been proposed as their origin in the case of HD 209458b (Tian et al. 2005). The warm Neptune GJ 436 also showed flux decreases of $15 \pm 2 \%$ between $30-110 \mathrm{~km} \mathrm{~s}^{-1}$. A colliding-wind scenario is unlikely to explain these redshifted signatures because they were observed between 0.5 and $6.6 \mathrm{~h}$ after the planetary transit (Lavie et al. 2017), and the planetary wind has a low density that favors charge-exchange in a collisionless exosphere (Bourrier et al. 2016). They could rather trace an accreting stream toward the star (Matsakos et al. 2015; Shaikhislamov et al. 2016; Khodachenko et al. 2017; Lavie et al. 2017).

\subsection{Atmospheric escape from GJ3470b}

Salz et al. (2016) performed 1D spherically symmetric simulations of the expanding thermosphere of GJ 3470b. They derived a heating efficiency on the order of $13 \%$, and predicted that the thermosphere should contain a large portion of neutral hydrogen, down to $\sim 50 \%$ at $4 R_{\mathrm{p}}$ and $\sim 20 \%$ at $8 R_{\mathrm{p}}$. Our simulations also suggest that large amounts of neutral hydrogen are present in the upper atmosphere of GJ 3470b, although at higher altitudes than in the simulations by Salz et al. (2016). Their results depend on the XUV and Lyman- $\alpha$ stellar irradiation, which they assumed to be about twice and four times higher than our observationally derived values. A lower irradiation in their simulations would increase the fraction of neutral hydrogen at a given altitude, which would be more consistent with our observations.

Our estimate for the intrinsic stellar Lyman- $\alpha$ ( $1.85 \mathrm{erg} \mathrm{cm}^{-2} \mathrm{~s}^{-1}$, after scaling to a stellar radius of $0.39 R_{\odot}$ ) and X-ray $\left(0.82 \mathrm{erg} \mathrm{cm}^{-2} \mathrm{~s}^{-1}\right)$ fluxes yields ages $\tau=2.1$ and $2.2 \mathrm{Gyr}$ from the M-dwarf relations in Guinan et al. (2016), respectively. The rotation period of GJ 3470 (20.7 days) further yields $\tau=1.9 \mathrm{Gyr}$ from the relation for early-type $\mathrm{M}$ dwarfs in Engle \& Guinan (2018). The consistency between the derived ages strengthens the reliability of these various relations. With the total mass-loss rate ranging between the neutral hydrogen mass-loss rate $\left(\sim 1.5 \times 10^{10} \mathrm{~g} \mathrm{~s}^{-1}\right)$ and the maximum energylimited mass-loss rate $\left(8.5 \times 10^{10} \mathrm{~g} \mathrm{~s}^{-1}\right)$, GJ 3470b would have lost between 1.1 and $6.5 \%$ of its current mass over its lifetime of $\sim 2$ Gyr. Because of a larger stellar XUV emission at the beginning of the star's life (e.g., Shkolnik \& Barman 2014), the total atmospheric escape over the planet lifetime is likely about five times stronger (Eq. 19 in Lecavelier des Etangs 2007), resulting in a mass loss of between $\sim 4$ and $35 \%$ of the total planet mass. A similar conclusion is reached when comparing the cumulative
XUV irradiation and escape velocity from GJ 3470b with that of solar system and extrasolar planets, as proposed by Zahnle \& Catling $(2017)^{2}$. GJ 3470b is located above their cosmic shoreline, that is, in a region where exoplanets might have lost a significant fraction of their atmosphere, and is predicted to have evaporated about $20 \%$ of its mass (assuming $\eta=20 \%$ ).

We can compare the upper atmospheric properties of GJ 3470b with those of GJ 436b, which is subjected to an XUV irradiation that is about three times lower (3938 vs $1303 \mathrm{erg} \mathrm{cm}^{-2} \mathrm{~s}^{-1}$, see Sect. 5 and Bourrier et al. 2016). Bourrier et al. (2016) constrained $\eta f_{H^{0}}$ to be on the order of $10^{-2}$ for GJ 436b, with a lower limit of $0.5 \%$ on both $\eta$ and $f_{H^{0}}$. A higher irradiation, combined with a lower heating efficiency and/or higher ionization fraction, likely explains why GJ 3470b today loses about a hundred times more neutral hydrogen than GJ 436b $\left(\sim 2.5 \times 10^{8} \mathrm{~g} \mathrm{~s}^{-1}\right)$. The mass-loss rate of the warm Neptune GJ 3470b is more in the range of theoretical and derived values for a hot Jupiter like HD 209458 (e.g., Bourrier \& Lecavelier des Etangs 2013), which is even subjected to a lower XUV irradiation (2929 $\mathrm{erg} \mathrm{cm}^{-2} \mathrm{~s}^{-1}$; Louden et al. 2017).

\subsection{Influence of radiation pressure and stellar wind}

In the present configuration of radiative blow-out (Bourrier \& Lecavelier des Etangs 2013), the velocity of exospheric atoms in the star rest frame is the combination of the orbital velocity of the planet at the time they escaped its gravitational influence (tangential to the orbital trajectory), and the velocity they then acquired under radiation pressure acceleration (in the opposite direction to the star). The velocity of a transiting atom projected on the LOS then determines the Doppler velocity of its absorption signature. The radial velocity of GJ 3470b increases from $-110 \mathrm{~km} \mathrm{~s}^{-1}$ near the approaching quadrature to about -5 to $15 \mathrm{~km} \mathrm{~s}^{-1}$ during transit. Thus, the planet contributes more to the radial velocity of particles escaping long before the transit. Furthermore, radiation pressure induces a similar dynamics on all escaping hydrogen atoms (neglecting self-shielding effects and the eccentricity of the GJ 3470 b orbit), but it takes up to $20 \mathrm{~h}$ to accelerate them to their terminal velocity (Fig. 12). Therefore, the radial velocity of a hydrogen atom at the time it transits the star depends on when it escaped the planet. Absorption is measured up to about $-90 \mathrm{~km} \mathrm{~s}^{-1}$, and simulations predict a peak of absorption at about $-60 \mathrm{~km} \mathrm{~s}^{-1}$ (Fig. 10). Figure 12 shows that neutral hydrogen atoms must escape the planet more than $5 \mathrm{~h}$ before its transit to occult the star with radial velocities higher than $-60 \mathrm{~km} \mathrm{~s}^{-1}$. However, the short photoionization lifetime $(\sim 1 \mathrm{~h}$, Sect. 5$)$ implies that a large portion of the neutral hydrogen atoms escaping from GJ $3470 \mathrm{~b}$ are ionized before they reach the observed velocities. Therefore, neutral hydrogen must escape in such large amounts from GJ 3470b to yield the absorption measured at high velocities in the blue wing.

The uncertainties on the reconstructed Lyman- $\alpha$ line profile only affect the radial velocity of neutral hydrogen atoms by a few $\mathrm{km} \mathrm{s}^{-1}$ during the transit, and thus have no significant impact on the simulations and our conclusions. Recent hydrodynamical simulations suggest that the outflow from the hot Jupiter HD 209458b is strongly collisional beyond the Roche lobe, preventing radiation pressure from imparting momentum to escaping neutral hydrogen atoms (Khodachenko et al. 2017; Cherenkov et al. 2018). Direct observations of the outflow at lower altitudes in the expanding thermosphere are required

\footnotetext{
2 We use the authors' conventions to place GJ 3470b in their Fig. 2, with an XUV irradiation of 230 times that of the Earth, and an escape velocity of $19 \mathrm{~km} \mathrm{~s}^{-1}$
} 


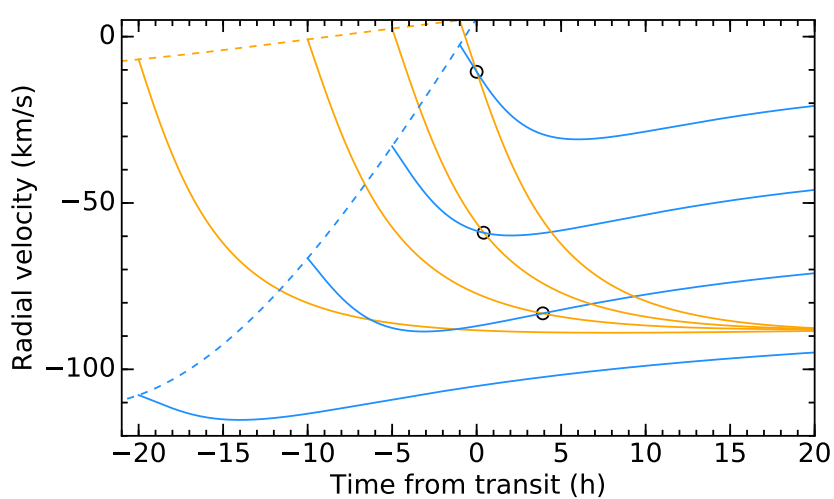

Fig. 12. Radial velocity of a neutral hydrogen atom escaping from GJ $3470 \mathrm{~b}$ as a function of time, projected on the star-atom axis (solid orange line) and on the LOS (solid blue line). The different curves correspond to four times of escape, which can be read at the intersection with the planet velocity curves (dashed lines). Black circles highlight the time of transit of the atoms.

to assess the validity of this scenario (Spake et al. 2018; Oklopčić \& Hirata 2018). Interestingly, however, all Lyman- $\alpha$ transit observations are well reproduced with a model of a collisionless exosphere accelerated by radiation pressure (Vidal-Madjar et al. 2003; Ehrenreich et al. 2012; Bourrier \& Lecavelier des Etangs 2013), combined in some cases with charge-exchange with the stellar wind (Lecavelier des Etangs et al. 2012; Bourrier \& Lecavelier des Etangs 2013; Bourrier et al. 2016; Lavie et al. 2017). Our present study of GJ 3470b shows that the blueshifted component of its Lyman- $\alpha$ absorption signature can be explained by a radiatively blown-out exosphere, without needing to invoke interactions with the stellar wind (Fig. 10). This contrasts with the planet GJ 436b, whose exosphere is shaped by the combination of radiative braking and charge-exchange with the low-velocity $\left(\sim 55 \mathrm{~km} \mathrm{~s}^{-1}\right)$ wind of its host star (Bourrier et al. 2016). Since GJ 3470 is an earlier-type, younger M dwarf than GJ 436 (which is older than 4 Gyr; Bourrier et al. 2018; Veyette \& Muirhead 2018), its stellar wind might be faster than the observable range of the stellar Lyman- $\alpha$ line $\left(\sim 200 \mathrm{~km} \mathrm{~s}^{-1}\right)$, which would explain why stellar wind protons neutralized by charge-exchange with the exosphere absorb too far in the wing to be detectable. Nonetheless, the wind from GJ 3470 could still have an influence on the upper atmosphere of its planet, for example, through the formation of a mixing layer (see Sect 6.3.1).

\section{Summary of findings and perspectives}

We observed the transit of the warm Neptune GJ 3470b in the Lyman- $\alpha$ line at three different epochs and obtained the following results:

1. We measure absorption depths of $35 \pm 7 \%$ in the blue wing of the line, between -94 and $-41 \mathrm{~km} \mathrm{~s}^{-1}$, and of $23 \pm 5 \%$ in the red wing, between 23 and $76 \mathrm{~km} \mathrm{~s}^{-1}$.

2. These absorption signatures are repeatable over the three epochs and are phased with the planet transit.

3. The redshifted absorption requires high densities of neutral hydrogen in the vicinity of the planet, in an elongated region extended up to $\sim 18 R_{\mathrm{p}}$ ahead of the planet and $\sim 27 R_{\mathrm{p}}$ behind it.

4. The blueshifted absorption is well explained by neutral hydrogen atoms escaping at a rate of $\sim 1.5 \times 10^{10} \mathrm{~g} \mathrm{~s}^{-1}$ and blown away from the star by radiation pressure. This may have led to the loss of $4-35 \%$ of the current mass of GJ 3470b over its $\sim 2$ Gyr lifetime.

GJ 3470b is the second warm Neptune after GJ 436b that is observed to evaporate and to be surrounded by an extended upper atmosphere of neutral hydrogen, supporting the idea that this class of exoplanets are ideal targets to be characterized through their upper atmosphere. GJ 3470b shows a higher mass loss and a smaller exosphere than GJ 436b. This likely results from the lower density of GJ 3470b, the stronger stellar energy input, photoionization, and radiation pressure from GJ 3470, and possibly from different wind properties. Observations of other escaping species not hindered by ISM absorption, such as helium at infrared wavelengths (Oklopčić \& Hirata 2018; Spake et al. 2018), would allow us to probe low-velocity regions in the upper atmosphere of these warm Neptunes to better understand their structure and formation. Further studies of these planets' present and past atmospheric properties are required to understand their presence at the edge of the evaporation desert, the role of their M-dwarf host stars, and the influence of their past dynamical evolution (see Bourrier et al. 2018 for GJ 436b).

Acknowledgements. We thank the referee for enlightening comments. We offer our thanks to Nathan Hara for his help with the random sampling of an ellipsoidal surface, and to Alexandre Correia for discussions about thermal tides. The research leading to these results received funding from the European Research Council under the European Union's Seventh Framework Program (FP7/20072013)/ERC grant agreement No. 336792. This work is based on observations made with the NASA/ESA HST (PanCET program, GO 14767), obtained at the Space Telescope Science Institute (STScI) operated by AURA, Inc. Support for this work was provided by NASA grants under the HST-GO-14767 program of the STScI. This work has been carried out in the frame of the National Centre for Competence in Research "PlanetS" supported by the Swiss National Science Foundation (SNSF). R.A. acknowledges the financial support of the SNSF. This project has received funding from the European Research Council (ERC) under the European Union's Horizon 2020 research and innovation programme (project Four Aces grant agreement No. 724427). A.L.E. acknowledges support from CNES and the French Agence Nationale de la Recherche (ANR), under programme ANR-12-BS05-0012 "Exo-Atmos". J.S.-F. acknowledges support from the Spanish MINECO grant AYA2016-79425-C3-2-P. This work has made use of data from the European Space Agency (ESA) mission Gaia (https: //www . cosmos.esa.int/gaia), processed by the Gaia Data Processing and Analysis Consortium (DPAC; https://www. cosmos.esa.int/web/gaia/dpac/ consortium). Funding for the DPAC has been provided by national institutions, in particular the institutions participating in the Gaia Multilateral Agreement.

\section{References}

Awiphan, S., Kerins, E., Pichadee, S., et al. 2016, MNRAS, 463, 2574

Beaugé, C., \& Nesvorný, D. 2013, ApJ, 763, 12

Ben-Jaffel, L. 2008, ApJ, 688, 1352

Ben-Jaffel, L., \& Sona Hosseini, S. 2010, ApJ, 709, 1284

Biddle, L. I., Pearson, K. A., Crossfield, I. J. M., et al. 2014, MNRAS, 443, 1810

Bisikalo, D. V., Kaigorodov, P. V., Ionov, D. E., \& Shematovich, V. I. 2013, Astron. Rep., 57, 715

Bonfils, X., Gillon, M., Udry, S., et al. 2012, A\&A, 546, A27

Bourrier, V., \& Lecavelier des Etangs, A. 2013, A\&A, 557, A124

Bourrier, V., Lecavelier des Etangs, A., Dupuy, H., et al. 2013, A\&A, 551, A63

Bourrier, V., Lecavelier des Etangs, A., \& Vidal-Madjar, A. 2014, A\&A, 565, A 105

Bourrier, V., Ehrenreich, D., \& Lecavelier des Etangs, A. 2015a, A\&A, 582, A65 Bourrier, V., Lecavelier des Etangs, A., \& Vidal-Madjar, A. 2015b, A\&A, 573, A11

Bourrier, V., Lecavelier des Etangs, A., Ehrenreich, D., Tanaka, Y. A., \& Vidotto, A. A. 2016, A\&A, 591, A121

Bourrier, V., Ehrenreich, D., Allart, R., et al. 2017a, A\&A, 602, A106

Bourrier, V., Ehrenreich, D., King, G., et al. 2017b, A\&A, 597, A26

Bourrier, V., Lovis, C., Beust, H., et al. 2018, Nature, 553, 477

Brown, T. M., Charbonneau, D., Gilliland, R. L., Noyes, R. W., \& Burrows, A. 2001, ApJ, 552, 699

Chen, G., Guenther, E. W., Pallé, E., et al. 2017, A\&A, 600, A138

Cherenkov, A. A., Bisikalo, D. V., \& Kosovichev, A. G. 2018, MNRAS, 475, 605

Crossfield, I. J. M., Barman, T., Hansen, B. M. S., \& Howard, A. W. 2013, A\&A, 559, A33 
Davis, T. A., \& Wheatley, P. J. 2009, MNRAS, 396, 1012

Demory, B.-O., Torres, G., Neves, V., et al. 2013, ApJ, 768, 154

Dragomir, D., Benneke, B., Pearson, K. A., et al. 2015, ApJ, 814, 102

Ehrenreich, D., \& Désert, J.-M. 2011, A\&A, 529, A136

Ehrenreich, D., Lecavelier des Etangs, A., Hébrard, G., et al. 2008, A\&A, 483, 933

Ehrenreich, D., Bourrier, V., Bonfils, X., et al. 2012, A\&A, 547, A18

Ehrenreich, D., Bonfils, X., Lovis, C., et al. 2014, A\&A, 570, A89

Ehrenreich, D., Bourrier, V., Wheatley, P. J., et al. 2015, Nature, 522, 459

Engle, S. G., \& Guinan, E. F. 2018, Res. Notes Am. Astron. Soc., 2, 34

Erkaev, N. V., Kulikov, Y. N., Lammer, H., et al. 2007, A\&A, 472, 329

Foreman-Mackey, D., Hogg, D. W., Lang, D., \& Goodman, J. 2013, PASP, 125 306

Fukui, A., Narita, N., Kurosaki, K., et al. 2013, ApJ, 770, 95

Fulton, B. J., Petigura, E. A., Howard, A. W., et al. 2017, AJ, 154, 109

Fulton, B. J., \& Petigura, E. A. 2018, AJ, accepted [arXiv:1805. 01453]

Gaia Collaboration (Prusti, T., et al.) 2016, A\&A, 595, A1

Gaia Collaboration (Brown, A. G. A., et al.) 2018, A\&A, 616, A1

García Muñoz, A. 2007, Planet. Space Sci., 55, 1426

Guinan, E. F., Engle, S. G., \& Durbin, A. 2016, ApJ, 821, 81

Guo, J. H., \& Ben-Jaffel, L. 2016, ApJ, 818, 107

Hébrard, G., \& Moos, H. W. 2003, ApJ, 599, 297

Hubbard, W. B., Hattori, M. F., Burrows, A., Hubeny, I., \& Sudarsky, D. 2007, Icarus, 187, 358

Huitson, C. M., Sing, D. K., Vidal-Madjar, A., et al. 2012, MNRAS, 422, 2477

Jin, S., \& Mordasini, C. 2018, ApJ, 853, 163

Jin, S., Mordasini, C., Parmentier, V., et al. 2014, ApJ, 795, 65

Johnstone, C. P., Güdel, M., Stökl, A., et al. 2015, ApJ, 815, L12

Khodachenko, M. L., Shaikhislamov, I. F., Lammer, H., et al. 2017, ApJ, 847, 126

Kislyakova, K. G., Holmström, M., Lammer, H., Odert, P., \& Khodachenko, M. L. 2014, Science, 346, 981

Kulow, J. R., France, K., Linsky, J., \& Loyd, R. O. P. 2014, ApJ, 786, 132

Kurokawa, H., \& Nakamoto, T. 2014, ApJ, 783, 54

Lammer, H., Selsis, F., Ribas, I., et al. 2003, ApJ, 598, L121

Lammer, H., Erkaev, N. V., Odert, P., et al. 2013, MNRAS, 430, 1247

Lavie, B., Ehrenreich, D., Bourrier, V., et al. 2017, A\&A, 605, L7

Lecavelier des Etangs, A. 2007, A\&A, 461, 1185

Lecavelier des Etangs, A., Vidal-Madjar, A., McConnell, J. C., \& Hébrard, G. 2004, A\&A, 418, L1

Lecavelier Des Etangs, A., Pont, F., Vidal-Madjar, A., \& Sing, D. 2008a, A\&A, 481, L83

Lecavelier Des Etangs, A., Vidal-Madjar, A., \& Désert, J.-M. 2008b, Nature, 456, E1

Lecavelier Des Etangs, A., Vidal-Madjar, A., Désert, J.-M., \& Sing, D. 2008c, A\&A, 485, 865

Lecavelier des Etangs, A., Ehrenreich, D., Vidal-Madjar, A., et al. 2010, A\&A, 514, A72

Lecavelier des Etangs, A., Bourrier, V., Wheatley, P. J., et al. 2012, A\&A, 543, L4
Liddle, A. R. 2007, MNRAS, 377, L74

Linsky, J. L., Draine, B. T., Moos, H. W., et al. 2006, ApJ, 647, 1106

Linsky, J. L., Bushinsky, R., Ayres, T., \& France, K. 2012, ApJ, 754, 69

Linsky, J. L., Fontenla, J., \& France, K. 2014, ApJ, 780, 61

Lopez, E. D., \& Fortney, J. J. 2013, ApJ, 776, 2

Lopez, E. D., Fortney, J. J., \& Miller, N. 2012, ApJ, 761, 59

Louden, T., Wheatley, P. J., \& Briggs, K. 2017, MNRAS, 464, 2396

Lundkvist, M. S., Kjeldsen, H., Albrecht, S., et al. 2016, Nat. Commun., 7, 11201

Matsakos, T., Uribe, A., \& Königl, A. 2015, A\&A, 578, A6

Mazeh, T., Holczer, T., \& Faigler, S. 2016, A\&A, 589, A75

Nascimbeni, V., Piotto, G., Pagano, I., et al. 2013, A\&A, 559, A32

Nikolov, N., Sing, D. K., Burrows, A. S., et al. 2015, MNRAS, 447, 463

Oklopčić, A., \& Hirata, C. M. 2018, ApJ, 855, L11

Owen, J. E., \& Alvarez, M. A. 2016, ApJ, 816, 34

Owen, J. E., \& Wu, Y. 2017, ApJ, 847, 29

Redfield, S., \& Linsky, J. L. 2008, ApJ, 673, 283

Salz, M., Czesla, S., Schneider, P. C., \& Schmitt, J. H. M. M. 2016, A\&A, 586, A75

Sanchis-Ojeda, R., Rappaport, S., Winn, J. N., et al. 2014, ApJ, 787, 47

Sanz-Forcada, J., \& Ribas, I. 2015, in Pathways Towards Habitable Planets, ASP Conf. Ser., 5

Sanz-Forcada, J., Micela, G., Ribas, I., et al. 2011, A\&A, 532, A6

Shaikhislamov, I. F., Khodachenko, M. L., Lammer, H., et al. 2016, ApJ, 832, 173

Shematovich, V. I., Ionov, D. E., \& Lammer, H. 2014, A\&A, 571, A94

Shkolnik, E. L., \& Barman, T. S. 2014, AJ, 148, 64

Sing, D. K., Vidal-Madjar, A., Désert, J.-M., Lecavelier des Etangs, A., \& Ballester, G. 2008, ApJ, 686, 658

Sing, D. K., Fortney, J. J., Nikolov, N., et al. 2016, Nature, 529, 59

Spake, J. J., Sing, D. K., Evans, T. M., et al. 2018, Nature, 557, 68 Strugarek, A. 2016, ApJ, 833, 140

Szabó, G. M., \& Kiss, L. L. 2011, ApJ, 727, L44

Tian, F., Toon, O. B., Pavlov, A. A., \& De Sterck H. 2005, ApJ, 621, 1049

Tremblin, P., \& Chiang, E. 2013, MNRAS, 428, 2565

Vernazza, J. E., Avrett, E. H., \& Loeser, R. 1981, ApJS, 45, 635

Veyette, M. J., \& Muirhead, P. S. 2018, ApJ, 863, 166

Vidal-Madjar, A., Lecavelier des Etangs, A., Désert, J.-M., et al. 2003, Nature, 422, 143

Vidal-Madjar, A., Désert, J.-M., Lecavelier des Etangs, A., et al. 2004, ApJ, 604, L69

Vidal-Madjar, A., Lecavelier des Etangs, A., Désert, J.-M., et al. 2008, ApJ, 676, L57

Vidotto, A. A., \& Bourrier, V. 2017, MNRAS, 470, 4026

Watson, A. J., Donahue, T. M., \& Walker, J. C. G. 1981, Icarus, 48, 150

Wood, B. E., Redfield, S., Linsky, J. L., Müller, H.-R., \& Zank, G. P. 2005, ApJS, 159, 118

Yelle, R. V. 2004, Icarus, 170, 167

Youngblood, A., France, K., Parke Loyd, R. O., et al. 2016, ApJ, 824, 101

Zahnle, K. J., \& Catling, D. C. 2017, ApJ, 843, 122 